(Supporting Information)

\title{
Titanium and Zirconium Complexes of Preorganized Tripodal Triaryloxide Ligands
}

\author{
Fumio Akagi, Tsukasa Matsuo and Hiroyuki Kawaguchi \\ Coordination Chemistry Laboratories, Institute for Molecular Science, \\ Myodaiji, Okazaki, 444-8787 Japan
}

\section{Contents}

Experimental Procedure

Table S1. Crystallographic Data for for 1a, 3, and 5.

Figure S1. Molecular Structure of 1a.

Table S2. Bond Distances $(\AA)$ for $\mathbf{1 a}$.

Table S3. Bond Angles (deg) for 1a.

Figure S2. Molecular Structure of 3.

Table S4. Bond Distances $(\AA)$ for 3 .

Table S5. Bond Angles (deg) for 3.

Figure S3. Molecular Structure of 5.

Table S6. Bond Distances $(\AA)$ for 5.

Table S7. Bond Angles (deg) for 5.

Figure S4. Kinetics plots of the isomerization of $\mathbf{1 a}$ into $\mathbf{1 b}$ in toluene- $d_{8}$.

Figure S5. Kinetics plots of the isomerization of $\mathbf{1 a}$ into $\mathbf{1 b}$ in 1,2-dichlorobenzene- $d_{4}$.

Figure S6. Arrhenius plot for the isomerization of $\mathbf{1 a}$ into $\mathbf{1 b}$ in toluene- $d_{8}$ (A) and in 1,2-dichlorobenzene- $d_{4}(\mathrm{~B})$. 


\section{Experimental Section}

General Considerations. Unless otherwise stated, all operations were performed either under an inert atmosphere of argon using standard Schlenk techniques or under nitrogen in an MBraun glove box. All dried solvents and chemicals commercially available were used as received without further purification. Benzene- $d_{6}$ and toluene- $d_{8}$ were dried and degassed over a potassium mirror, and vacuum transferred prior to use. $\quad \mathrm{H}_{3}\left[\mathbf{O}_{3}\right]$ (= tris(3,5-di-tert-butyl-2-hydroxyphenyl)methane), $\mathrm{Ti}\left(\mathrm{NEt}_{2}\right)_{4}$, and $\mathrm{Zr}\left(\mathrm{CH}_{2} \mathrm{Ph}\right)_{4}$ were prepared by a literature procedure. All other chemicals were used as received. NMR spectra were recorded on JEOL LA-400 spectrometer. ${ }^{1} \mathrm{H}$ and ${ }^{13} \mathrm{C}$ NMR are reported with reference to solvent resonances (residual $\mathrm{C}_{6} \mathrm{D}_{5} \mathrm{H}$ in $\mathrm{C}_{6} \mathrm{D}_{6}, \delta 7.15$ and $\delta 128.0$; the methyl group of $\mathrm{C}_{7} \mathrm{D}_{7} \mathrm{H}$ in $\mathrm{C}_{7} \mathrm{D}_{8}, \delta$ 2.09). Elemental analyses were measured using Yanaco MT-6 and MSU-32 microanalyzers.

Synthesis of $\left[s y n-\mathrm{O}_{3}\right] \mathrm{Ti}\left(\mathbf{N E t}_{2}\right)$ (1a). A toluene solution of $\operatorname{Ti}\left(\mathrm{NEt}_{2}\right)_{4}(7.7 \mathrm{~mL}, 0.26 \mathrm{M}, 2.01$ mmol) was added to a suspension of $\mathrm{H}_{3}\left[\mathbf{O}_{3}\right](1.26 \mathrm{~g}, 2.01 \mathrm{mmol})$ in toluene $(30 \mathrm{~mL})$ at room temperature. The mixture was stirred for $3 \mathrm{~h}$ to give an orange homogeneous solution, and then all volatiles were removed in vacuo. The residue was crystallized from hexane, yielding 1a as orange crystals in 76\% (1.13 g). $\quad{ }^{1} \mathrm{H}$ NMR (400 MHz, benzene- $d_{6}$ at rt): $1.22\left(\mathrm{t}, 6 \mathrm{H}, J=7.0 \mathrm{~Hz}, \mathrm{CH}_{3}\right), 1.34$ (s, 27H, $\left.{ }^{\mathrm{t}} \mathrm{Bu}\right), 1.49\left(\mathrm{~s}, 27 \mathrm{H},{ }^{\mathrm{t}} \mathrm{Bu}\right), 3.91\left(\mathrm{q}, 4 \mathrm{H}, J=7.0 \mathrm{~Hz}, \mathrm{CH}_{2}\right), 4.96(\mathrm{~s}, 1 \mathrm{H}, \mathrm{CH}), 7.30(\mathrm{~d}, 3 \mathrm{H}, J=2.4 \mathrm{~Hz}$, $\mathrm{Ar}), 8.00(\mathrm{~d}, 3 \mathrm{H}, J=2.4 \mathrm{~Hz}, \mathrm{Ar}) .{ }^{13} \mathrm{C} \mathrm{NMR}(100 \mathrm{MHz}): 15.8\left(\mathrm{CH}_{3}\right), 30.0,31.7,34.5,35.7\left({ }^{\mathrm{t}} \mathrm{Bu}\right), 45.9$ $\left(\mathrm{d},{ }^{1} J_{\mathrm{CH}}=91.1 \mathrm{~Hz}, \mathrm{CH}\right), 46.2\left(\mathrm{CH}_{2}\right), 122.5,122.7,136.0,139.3,144.0,163.5$ (Ar). Anal. Calcd for $\mathrm{C}_{47} \mathrm{H}_{71} \mathrm{O}_{3} \mathrm{NTi}: \mathrm{C}, 75.68 ; \mathrm{H}, 9.59 ; \mathrm{N}, 1.88$. Found: C, 75.16; H, 9.60; N, 1.88 .

Synthesis of $\left[\right.$ anti- $\left._{3}\right] \mathbf{T i}\left(\mathbf{N E t}_{2}\right)$ (1b). Complex 1a $(21 \mathrm{mg}, 0.028 \mathrm{mmol})$ was dissolved in benzene- $d_{6}(0.6 \mathrm{ml})$ in a $\mathrm{J}$-Young NMR tube and was quantitatively transformed into $\mathbf{1 b}$ while being kept at $60^{\circ} \mathrm{C}$ for 13 days. ${ }^{1} \mathrm{H}$ NMR (400 MHz, benzene- $d_{6}$ at $\left.\mathrm{rt}\right): 1.10$ (t, $\left.6 \mathrm{H} . J=7.0 \mathrm{~Hz}, \mathrm{CH}_{3}\right), 1.19(\mathrm{~s}$, 27H, tBu), $1.46(\mathrm{~s}, 27 \mathrm{H}, \mathrm{tBu}), 3.91\left(\mathrm{q}, 4 \mathrm{H}, J=7.0 \mathrm{~Hz}, \mathrm{CH}_{2}\right), 6.04(\mathrm{~s}, 1 \mathrm{H}, \mathrm{CH}), 7.38(\mathrm{~d}, 3 \mathrm{H}, J=2.4 \mathrm{~Hz}$, Ar), $7.54(\mathrm{~d}, 3 \mathrm{H}, J=2.4 \mathrm{~Hz}, \mathrm{Ar}) .{ }^{13} \mathrm{C} \mathrm{NMR}(100 \mathrm{MHz}) \quad 14.9\left(\mathrm{CH}_{3}\right), 30.5,31.7,34.4,35.6\left({ }^{\mathrm{t}} \mathrm{Bu}\right)$, 
$44.9\left(\mathrm{CH}_{2}\right), 64.1\left(\mathrm{~d},{ }^{1} J_{\mathrm{CH}}=122.3 \mathrm{~Hz}, \mathrm{CH}\right), 123.6,129.0,135.9,136.1,144.0,161.1(\mathrm{Ar})$.

Synthesis of $\left[\right.$ anti- $\left.\mathbf{O}_{3}\right]$ TiCl (2). A J-Young NMR tube was charged with a benzene- $d_{6}$ solution $(0.6 \mathrm{ml})$ of $1 \mathrm{a}(4.6 \mathrm{mg}, 6.2 \mu \mathrm{mol})$ and $\mathrm{Me}_{3} \mathrm{SiCl}(0.8 \mu \mathrm{L}, 6.3 \mu \mathrm{mol})$. The orange solution was allowed to stand at room temperature overnight. The ${ }^{1} \mathrm{H}$ NMR spectrum of the resulting mixture showed only resonances attributable to 2 and $\mathrm{Me}_{3} \mathrm{SiNEt}_{2} . \quad{ }^{1} \mathrm{H} \mathrm{NMR}\left(400 \mathrm{MHz}\right.$, benzene- $d_{6}$ at $\left.\mathrm{rt}\right): 1.13(\mathrm{~s}, 27 \mathrm{H}, \mathrm{tBu})$, $1.43(\mathrm{~s}, 27 \mathrm{H}, \mathrm{tBu}), 5.89(\mathrm{~s}, 1 \mathrm{H}, \mathrm{CH}), 7.33(\mathrm{~d}, 3 \mathrm{H} J=2.4 \mathrm{~Hz}, \mathrm{Ar}), 7.50(\mathrm{~d}, J=2.4 \mathrm{~Hz}, \mathrm{Ar}) . \quad{ }^{13} \mathrm{C} \mathrm{NMR}$ (100 MHz): 30.4, 31.4, 34.5, $35.5\left({ }^{\mathrm{t}} \mathrm{Bu}\right), 62.9\left(\mathrm{~d},{ }^{1} J_{\mathrm{CH}}=122.6 \mathrm{~Hz}, C \mathrm{H}\right), \quad 123.9,128.4,135.9,136.7$, 146.5, 163.8 (Ar). Anal. Calcd for $\mathrm{C}_{43} \mathrm{H}_{61} \mathrm{O}_{3} \mathrm{ClTi} \bullet\left(\mathrm{C}_{7} \mathrm{H}_{8}\right)_{2.5}: \mathrm{C}, 77.33 ; \mathrm{H}, 8.69$. Found: C, 77.08; H, 8.79 .

Synthesis of $\left[\right.$ anti- $\left.\mathbf{O}_{3}\right] \mathbf{T i}\left(\mathbf{C H}_{2} \mathbf{P h}\right)(3)$. To a solution of $2(727 \mathrm{mg}, 0.738 \mathrm{mmol})$ in toluene (40 ml) was slowly added 1.0 M solution of $\mathrm{PhCH}_{2} \mathrm{MgCl}$ in diethyl ether $(0.82 \mathrm{ml}, 0.82 \mathrm{mmol})$ at $-98{ }^{\circ} \mathrm{C}$. The mixture was allowed to warm up to room temperature and was stirred for $6 \mathrm{~h}$. Removal of the solvent in vacuo, extraction of the residue with hexane, and centrifugation yielded an orange solution. The solution was concentrated to afford a yellow powder of 3 in $74 \%$ (416 mg). ${ }^{1} \mathrm{H} \mathrm{NMR}(400 \mathrm{MHz}$, benzene- $d_{6}$ at rt): $1.15\left(\mathrm{~s}, 27 \mathrm{H},{ }^{\mathrm{t}} \mathrm{Bu}\right), 1.39\left(\mathrm{~s}, 27 \mathrm{H},{ }^{\mathrm{t}} \mathrm{Bu}\right), 3.26\left(\mathrm{~s}, 2 \mathrm{H}, \mathrm{CH}_{2}\right), 5.89(\mathrm{~s}, 1 \mathrm{H}, \mathrm{CH}), 6.94(\mathrm{t}, J=$ $7.3 \mathrm{~Hz}, 1 \mathrm{H}, \mathrm{ArH}), 7.18(\mathrm{t}, J=7.3 \mathrm{~Hz}, 2 \mathrm{H}, \mathrm{ArH}), 7.31(\mathrm{~d}, J=7.3 \mathrm{~Hz}, 2 \mathrm{H}, \operatorname{ArH}), 7.35(\mathrm{~d}, J=2.3 \mathrm{~Hz}, 3 \mathrm{H}$, $\operatorname{ArH}), 7.46(\mathrm{~d}, J=2.3 \mathrm{~Hz}, 3 \mathrm{H}, \mathrm{ArH}) .{ }^{13} \mathrm{C}(100 \mathrm{MHz}): 30.5,31.5,34.4,35.5\left({ }^{\mathrm{t}} \mathrm{Bu}\right), 64.0\left(\mathrm{~d},{ }^{1} J_{\mathrm{CH}}=\right.$ 121.1 Hz, $C H$ ), $78.6\left(\mathrm{t},{ }^{1} J_{\mathrm{CH}}=124.3 \mathrm{~Hz}, C_{2}\right), 123.9,129.4,135.9,136.0,145.0,160.9$ (Ar). Anal. Calcd for $\mathrm{C}_{50} \mathrm{H}_{68} \mathrm{O}_{3}$ Ti: C, 78.51; H, 8.96. Found: C, 78.03; H, 9.13.

Reaction of $\mathbf{H}_{3}\left[\mathbf{O}_{3}\right]$ with $\mathbf{Z r}\left(\mathbf{C H}_{2} \mathbf{P h}\right)_{4}$. A mixture of $\mathrm{H}_{3}\left[\mathbf{O}_{3}\right](332 \mathrm{mg}, 0.53 \mathrm{mmol})$ and $\mathrm{Zr}\left(\mathrm{CH}_{2} \mathrm{Ph}\right)_{4}(242 \mathrm{mg}, 0.53 \mathrm{mmol})$ was dissolved in toluene $(8 \mathrm{ml})$ containing a small amount of THF $(0.1 \mathrm{ml})$. The mixture was stirred for $1 \mathrm{~h}$ to give a yellow homogeneous solution, and then all volatiles were removed in vacuo to leave a yellow solid. The NMR data show that the resulting solid contained $\left[\right.$ syn- $\left.\mathbf{O}_{3}\right] \mathrm{Zr}\left(\mathrm{CH}_{2} \mathrm{Ph}\right)(\mathbf{4})$ and $\left[\mathbf{O}_{3} \mathbf{C}\right] \mathrm{Zr}(\mathrm{THF})_{3}(\mathbf{5})$ in a ca. 3:1 ratio. The residue was dissolved 
in toluene and stirred for $12 \mathrm{~h}$ at $60{ }^{\circ} \mathrm{C}$. After the solution was evaporated to dryness, the residue was washed with pentane to give $\mathbf{5}$ as pale yellow powder (194 mg, $39 \%$ yield).

Data for 4: ${ }^{1} \mathrm{H}$ NMR (400 MHz, benzene- $d_{6}$ at rt): 1.20 (br, THF), $1.44(\mathrm{~s}, 27 \mathrm{H}, \mathrm{tBu}), 1.54$ (s, 27H, tBu), $2.86\left(\mathrm{~s}, 2 \mathrm{H}, \mathrm{CH}_{2}\right), 3.73(\mathrm{br}, \mathrm{THF}), 5.79(\mathrm{~s}, 1 \mathrm{H}, \mathrm{CH}), 6.88(\mathrm{t}, J=7.4 \mathrm{~Hz}, 1 \mathrm{H}, \mathrm{Ar} \mathrm{H}), 7.22(\mathrm{t}, J$ $=7.4 \mathrm{~Hz}, 2 \mathrm{H}, \mathrm{Ar} \mathrm{H}), 7.28(\mathrm{~d}, J=7.4 \mathrm{~Hz}, 2 \mathrm{H}, \mathrm{Ar} \mathrm{H}), 7.41(\mathrm{~d}, J=2.2 \mathrm{~Hz}, 3 \mathrm{H}, \mathrm{Ar}), 8.26(\mathrm{~d}, J=2.2 \mathrm{~Hz}$,

3H, Ar). ${ }^{13} \mathrm{C} \mathrm{NMR}(100 \mathrm{MHz}): 25.2$ (THF), 30.9, 31.9, 34.5, $35.8\left({ }^{\mathrm{t}} \mathrm{Bu}\right), 40.5\left(\mathrm{~d},{ }^{1} J_{\mathrm{CH}}=96.8 \mathrm{~Hz}, \mathrm{CH}\right)$, $62.0\left(\mathrm{t},{ }^{1} J_{\mathrm{CH}}=114.0 \mathrm{~Hz}, C \mathrm{H}_{2}\right), 71.4(\mathrm{THF}), 120.2,121.9,122.5,125.8,135.7,139.5,141.4,151.8$, 160.9 (Ar).

Data for 5: ${ }^{1} \mathrm{H}$ NMR (400 MHz, benzene- $d_{6}$ at rt): 1.36 (br, 12H, THF), $1.48(\mathrm{~s}, 27 \mathrm{H}, \mathrm{tBu}), 1.55$ (s, 27H, tBu), 3.83 (br, 12H, THF), $7.34(\mathrm{~d}, J=2.2 \mathrm{~Hz}, 3 \mathrm{H}, \mathrm{Ar}), 7.97(\mathrm{~d}, J=2.2 \mathrm{~Hz}, 3 \mathrm{H}, \mathrm{Ar}) .{ }^{13} \mathrm{C}$ NMR (100 MHz): 25.2 (THF), 35.0, 34.6, 32.3, 31.2 ('Bu), 71.0 (THF), 83.6 (C), 120.0, 132.1, 139.6, 148.0, 164.8 (Ar). Anal. Calcd for $\mathrm{C}_{55} \mathrm{H}_{84} \mathrm{O}_{6} \mathrm{Zr}: \mathrm{C}, 70.84 ; \mathrm{H}, 9.08$. Found: C, 69.65; H, 9.11.

Kinetic Analysis of the Conversion of the syn-Complex 1a to the anti-Complex 1b. The conversion of $\mathbf{1 a}$ to $\mathbf{1 b}$ was conducted in sealed tubes, and the concentration of the product was monitored periodically by ${ }^{1} \mathrm{H}$ NMR in toluene- $d_{8}$ and in 1,2-dichlorobenzene- $d_{4}$. The data were treated in terms of first-order kinetics: plots of $\ln [x / a]$ ( $a$, the initial concentration of 1a; $x$, the concentration of 1a at time $t$ ) vs time resulted in straight lines (Figure S4, S5). The rate constants $k$ were extracted as the slopes of these lines. The $k\left(\mathrm{~s}^{-1}\right)$ values obtained at give temperatures are as follows; in toluene- $d_{8}, 3.18(6) \times 10^{-6}, 337 \mathrm{~K} ; 1.13(2) \times 10^{-5}, 353 \mathrm{~K} ; 2.14(4) \times 10^{-5}, 364 \mathrm{~K} ; 3.81(6) \times 10^{-5}$, $373 \mathrm{~K}$; in 1,2-dichlorobenzene- $d_{4}, 1.61(5) \times 10^{-6}, 337 \mathrm{~K}$; 9.91(6) x $10^{-6}, 363 \mathrm{~K}$; $2.16(7) \times 10^{-5}, 373 \mathrm{~K}$; $6.09(15) \times 10^{-5}, 393 \mathrm{~K}$. Activation parameters were determined from Arrhenius plots $\left(R^{2}>0.99\right)$ (Figure S6).

Crystal Structure Determination. Crystallographic data for 1a, 3, and $\mathbf{5}$ are summarized in Table S1. Single crystals of $\mathbf{1 a}$ were obtained form a toluene solution, while those of $\mathbf{3}$ were obtained from a hexane solution. The slow diffusion of hexamethyldisiloxane into a toluene solution of $\mathbf{5}$ 
afforded single crystals. Crystals of these complexes immersed in mineral oil on nylon loops and transferred to a Rigaku Mercury CCD system (for 1a and 3) or Saturn CCD system (for 5) equipped with a Rigaku GNNP low-temperature device. Data were collected under a cold nitrogen stream (173 K) using graphite-monochromated Mo $K \alpha$ radiation $(\lambda=0.71070 \AA)$. Equivalent reflections were merged, and the images were processed with the CrystalClear (Rigaku) program. Corrections for Lorentz-polarization effects and absorption were performed.

Calculations were performed with the CrystalStructure (Rigaku) software package. The structures were solved by direct methods, and the remaining heavy atoms were found in subsequent Fourier maps. The structures were refined on $F^{2}$ by the full-matrix least-squares method.

The molecule of $\mathbf{1 a}$ are bisected by a crystallographic mirror plane containing Ti, N, O(1), C(1), $\mathrm{C}(2), \mathrm{C}(3), \mathrm{C}(4), \mathrm{C}(5), \mathrm{C}(6), \mathrm{C}(7), \mathrm{C}(15), \mathrm{C}(16), \mathrm{C}(18), \mathrm{C}(19)$, and $\mathrm{H}(1)$. One of the tert-butyl groups was rotationally disordered with the typical fashion and modeled as six carbon atoms, while the ethyl groups of the amide was disordered over two positions. In this compound 1a, the residual electron density near the titanium atom is high. To solve this problem, different crystals were measured. Despite very careful measurements, the large difference peak remains. Because absorption corrections or no correction at all does not have any effect, it is probably a genuine disorder effect. The carbon atoms of the disordered tert-butyl group of 1a were refined isotropically, whereas the remaining non-hydrogen atoms were refined anisotropically. The methine proton of the ligand in 1a was located and refined isotropically. All hydrogen atoms except for the methine protons were put at calculated positions with $\mathrm{C}-\mathrm{H}$ distances of $0.97 \AA$. In the case of 1a, systemtic absences were consistent with $C m c 2_{1}$ (\#36), Ama2 (\#40), and Cmcm (\#63) space groups. The choice of the acentric space group $C m c 2_{1}$ was confirmed by the successful refinement of the model. The Flack parameter refined to $0.06(2)$, indicating that the correct absolute structure was used.

For 3, the asymmetric unit contains two crystallographically independent molecules. The benzyl group of each independent molecule in $\mathbf{3}$ was disordered, as evidenced by large thermal motion, but multiple sites were not found for the constituent atoms. All non-hydrogen atoms were refined anisotropically. The methine proton of the ligand in each independent molecule was located and 
refined isotropically. All hydrogen atoms except for the methine protons were put at calculated positions with $\mathrm{C}-\mathrm{H}$ distances of $0.97 \AA$.

For 5, the methylene of one THF molecule was disordered over two positions. All non-hydrogen atoms were refined anisotropically. The crystals of $\mathbf{5}$ contained the solvent molecules (toluene), and their thermal motion was relatively large. All hydrogen atoms were put at calculated positions with $\mathrm{C}-\mathrm{H}$ distances of $0.97 \AA$, where no hydrogen atom of the distorted methylene carbon and its adjacent carbon atoms in the distorted THF molecule was included.

Additional crystallographic data are given in the cif files. 
Table S1. Crystallographic Data for 1a, 3, and 5.

\begin{tabular}{|c|c|c|c|}
\hline & $\mathbf{1 a}$ & 3 & 5 \\
\hline formula & $\mathrm{C}_{47} \mathrm{H}_{71} \mathrm{O}_{3} \mathrm{NTi}$ & $\mathrm{C}_{50} \mathrm{H}_{68} \mathrm{O}_{3} \mathrm{Ti}$ & $\mathrm{C}_{60} \mathrm{H}_{92} \mathrm{O}_{6} \mathrm{Zr}$ \\
\hline$M$ & 745.98 & 764.99 & 1024.63 \\
\hline$T / \mathrm{K}$ & 173 & 173 & 173 \\
\hline color & orange & yellow & yellow \\
\hline crystal size, $\mathrm{mm}$ & $0.20 \times 0.20 \times 0.20$ & $0.15 \times 0.30 \times 0.30$ & $0.10 \times 0.20 \times 0.20$ \\
\hline crystal system & orthorhombic & triclinic & monoclinic \\
\hline space group & $C m c 2_{1}$ (\# 36) & $P 1$ (bar) (\# 2) & $P 2_{1} / n(\# 14)$ \\
\hline$a / \AA$ & $18.380(6)$ & $10.932(6)$ & $15.589(4)$ \\
\hline$b / \AA$ & $24.348(8)$ & $18.807(10)$ & $18.322(4)$ \\
\hline$c / \AA$ & $10.254(3)$ & $22.851(12)$ & $20.355(5)$ \\
\hline$\alpha / \operatorname{deg}$ & & $95.696(7)$ & \\
\hline$\beta / \mathrm{deg}$ & & $97.034(9)$ & $93.609(4)$ \\
\hline$\gamma / \operatorname{deg}$ & & $96.286(6)$ & \\
\hline$V / \AA$ & $4588.7(25)$ & $4604.1(41)$ & $5802.1(24)$ \\
\hline$Z$ & 4 & 4 & 4 \\
\hline$D c / \mathrm{g} \mathrm{cm}^{-3}$ & 1.080 & 1.104 & 1.173 \\
\hline$\mu(\mathrm{Mo}-\mathrm{K} \alpha) / \mathrm{cm}^{-1}$ & 2.23 & 2.24 & 2.37 \\
\hline reflections collected & 36116 & 71158 & 89872 \\
\hline unique reflections & 2836 & 19588 & 13601 \\
\hline refined parameters & 312 & 1115 & 717 \\
\hline GOF on $F^{2}$ & 1.002 & 1.001 & 1.00 \\
\hline$R 1[I>2 \sigma(I)]^{\mathrm{a}}$ & 0.063 & 0.074 & 0.076 \\
\hline $\mathrm{w} R 2$ (all data) $^{\mathrm{b}}$ & 0.181 & 0.244 & 0.239 \\
\hline Flack parameter & $0.05(2)$ & & \\
\hline
\end{tabular}


Figure S1. Molecular Structure of 1a.

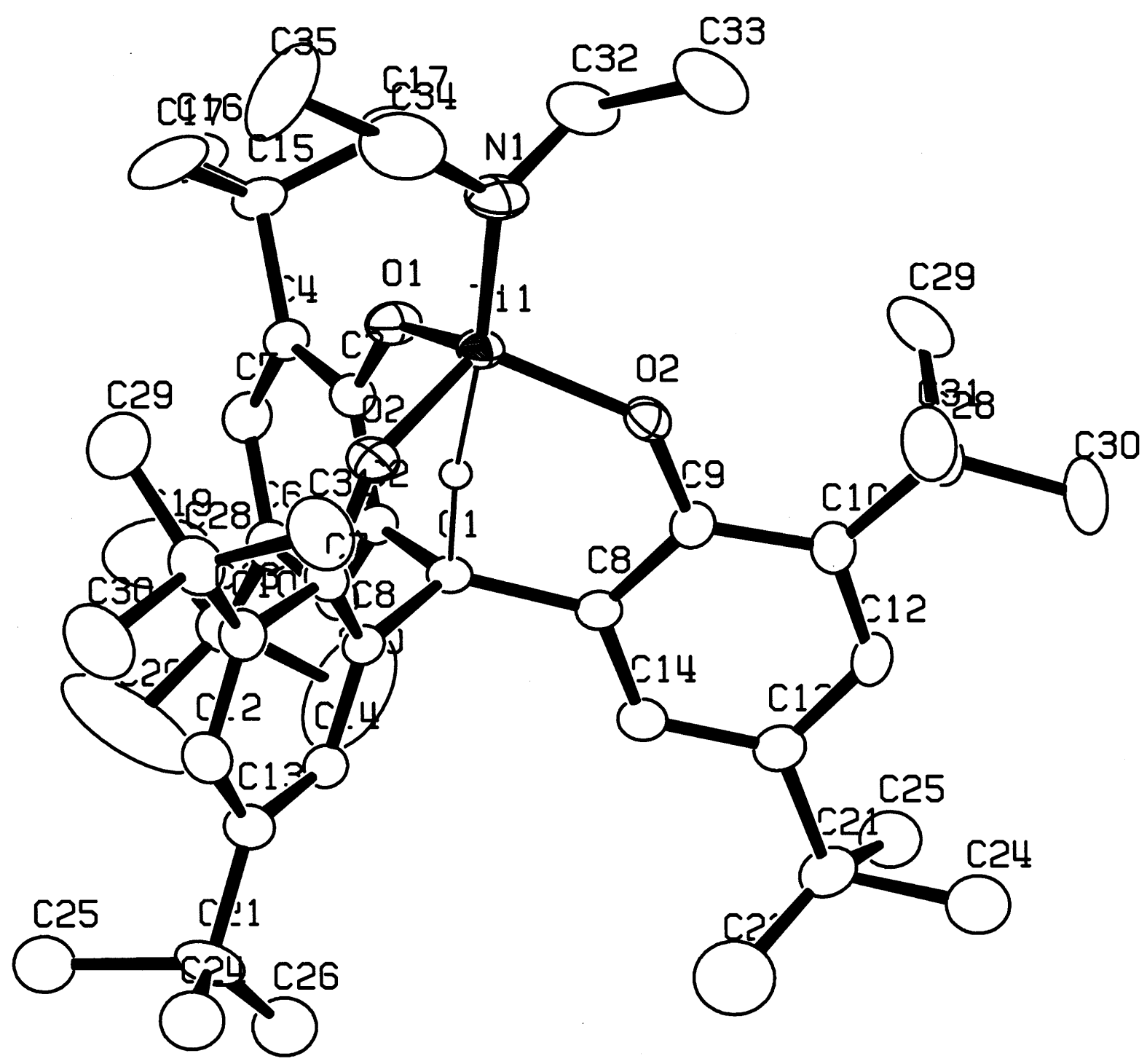


Table S2. Bond Distances $(\AA)$ for $\mathbf{1 a}$.

\begin{tabular}{llllll}
\hline atom & atom & distance & atom & atom & distance \\
& & & & & \\
$\mathrm{Ti}(1)$ & $\mathrm{O}(1)$ & $1.852(2)$ & $\mathrm{Ti}(1)$ & $\mathrm{O}(2)$ & $1.853(1)$ \\
$\mathrm{Ti}(1)$ & $\mathrm{N}(1)$ & $1.864(2)$ & $\mathrm{O}(1)$ & $\mathrm{C}(3)$ & $1.376(3)$ \\
$\mathrm{O}(2)$ & $\mathrm{C}(9)$ & $1.378(2)$ & $\mathrm{N}(1)$ & $\mathrm{C}(32)$ & $1.428(6)$ \\
$\mathrm{N}(1)$ & $\mathrm{C}(34)$ & $1.494(6)$ & $\mathrm{C}(1)$ & $\mathrm{C}(2)$ & $1.533(3)$ \\
$\mathrm{C}(1)$ & $\mathrm{C}(8)$ & $1.524(2)$ & $\mathrm{C}(2)$ & $\mathrm{C}(3)$ & $1.406(3)$ \\
$\mathrm{C}(2)$ & $\mathrm{C}(7)$ & $1.382(3)$ & $\mathrm{C}(3)$ & $\mathrm{C}(4)$ & $1.413(3)$ \\
$\mathrm{C}(4)$ & $\mathrm{C}(5)$ & $1.396(3)$ & $\mathrm{C}(4)$ & $\mathrm{C}(15)$ & $1.552(3)$ \\
$\mathrm{C}(5)$ & $\mathrm{C}(6)$ & $1.402(3)$ & $\mathrm{C}(6)$ & $\mathrm{C}(7)$ & $1.403(3)$ \\
$\mathrm{C}(6)$ & $\mathrm{C}(18)$ & $1.536(3)$ & $\mathrm{C}(8)$ & $\mathrm{C}(9)$ & $1.402(2)$ \\
$\mathrm{C}(8)$ & $\mathrm{C}(14)$ & $1.374(2)$ & $\mathrm{C}(9)$ & $\mathrm{C}(10)$ & $1.411(2)$ \\
$\mathrm{C}(10)$ & $\mathrm{C}(12)$ & $1.390(2)$ & $\mathrm{C}(10)$ & $\mathrm{C}(28)$ & $1.542(2)$ \\
$\mathrm{C}(12)$ & $\mathrm{C}(13)$ & $1.398(2)$ & $\mathrm{C}(13)$ & $\mathrm{C}(14)$ & $1.386(2)$ \\
$\mathrm{C}(13)$ & $\mathrm{C}(21)$ & $1.534(2)$ & $\mathrm{C}(15)$ & $\mathrm{C}(16)$ & $1.507(4)$ \\
$\mathrm{C}(15)$ & $\mathrm{C}(17)$ & $1.516(3)$ & $\mathrm{C}(18)$ & $\mathrm{C}(19)$ & $1.489(4)$ \\
$\mathrm{C}(18)$ & $\mathrm{C}(20)$ & $1.512(4)$ & $\mathrm{C}(21)$ & $\mathrm{C}(22)$ & $1.499(8)$ \\
$\mathrm{C}(21)$ & $\mathrm{C}(23)$ & $1.619(6)$ & $\mathrm{C}(21)$ & $\mathrm{C}(24)$ & $1.686(6)$ \\
$\mathrm{C}(21)$ & $\mathrm{C}(25)$ & $1.675(5)$ & $\mathrm{C}(21)$ & $\mathrm{C}(26)$ & $1.557(4)$ \\
$\mathrm{C}(21)$ & $\mathrm{C}(27)$ & $1.477(6)$ & $\mathrm{C}(22)$ & $\mathrm{C}(25)$ & $0.529(8)$ \\
$\mathrm{C}(23)$ & $\mathrm{C}(26)$ & $0.957(7)$ & $\mathrm{C}(24)$ & $\mathrm{C}(27)$ & $0.492(8)$ \\
$\mathrm{C}(28)$ & $\mathrm{C}(29)$ & $1.549(3)$ & $\mathrm{C}(28)$ & $\mathrm{C}(30)$ & $1.534(3)$ \\
$\mathrm{C}(28)$ & $\mathrm{C}(31)$ & $1.532(3)$ & $\mathrm{C}(32)$ & $\mathrm{C}(33)$ & $1.540(9)$ \\
$\mathrm{C}(34)$ & $\mathrm{C}(35)$ & $1.399(9)$ & $\mathrm{C}(1)$ & $\mathrm{H}(1)$ & $1.19(2)$ \\
$\mathrm{Ti}(1)$ & $\mathrm{H}(1)$ & $1.66(2)$ & & & \\
& & & & &
\end{tabular}


Table S3. Bond Angles (deg) for 1a.

\begin{tabular}{|c|c|c|c|c|c|c|c|}
\hline atom & atom & atom & angle & atom & atom & atom & angle \\
\hline $\mathrm{O}(1)$ & $\operatorname{Ti}(1)$ & $\mathrm{O}(2)$ & $105.75(5)$ & $\mathrm{O}(1)$ & $\operatorname{Ti}(1)$ & $\mathrm{N}(1)$ & $108.53(8)$ \\
\hline $\mathrm{O}(2)$ & $\operatorname{Ti}(1)$ & $\mathrm{N}(1)$ & $112.68(5)$ & $C(3)$ & $\mathrm{O}(1)$ & $\operatorname{Ti}(1)$ & $136.6(1)$ \\
\hline $\mathrm{C}(9)$ & $\mathrm{O}(2)$ & $\operatorname{Ti}(1)$ & $136.4(1)$ & $C(32)$ & $\mathrm{N}(1)$ & $\mathrm{C}(34)$ & $115.3(3)$ \\
\hline$C(32)$ & $\mathrm{N}(1)$ & $\operatorname{Ti}(1)$ & $116.1(2)$ & $C(34)$ & $\mathrm{N}(1)$ & $\operatorname{Ti}(1)$ & $126.4(2)$ \\
\hline $\mathrm{C}(2)$ & $\mathrm{C}(1)$ & $\mathrm{C}(8)$ & $117.3(1)$ & $\mathrm{C}(3)$ & $\mathrm{C}(2)$ & $C(7)$ & $120.6(2)$ \\
\hline $\mathrm{C}(3)$ & $C(2)$ & $\mathrm{C}(1)$ & $115.8(2)$ & $C(7)$ & $C(2)$ & $\mathrm{C}(1)$ & $123.7(2)$ \\
\hline$C(4)$ & $\mathrm{C}(3)$ & $\mathrm{O}(1)$ & $119.8(2)$ & $C(4)$ & $\mathrm{C}(3)$ & $\mathrm{C}(2)$ & $120.2(2)$ \\
\hline $\mathrm{O}(1)$ & $\mathrm{C}(3)$ & $\mathrm{C}(2)$ & $120.0(2)$ & $C(5)$ & $\mathrm{C}(4)$ & $\mathrm{C}(15)$ & $122.1(2)$ \\
\hline $\mathrm{C}(5)$ & $C(4)$ & $\mathrm{C}(3)$ & $117.6(2)$ & $\mathrm{C}(15)$ & $C(4)$ & $\mathrm{C}(3)$ & $120.4(2)$ \\
\hline$C(6)$ & $C(5)$ & $C(4)$ & $123.1(2)$ & $C(7)$ & $C(6)$ & $\mathrm{C}(18)$ & $121.0(2)$ \\
\hline$C(7)$ & $C(6)$ & $C(5)$ & $117.8(2)$ & $\mathrm{C}(18)$ & $\mathrm{C}(6)$ & $\mathrm{C}(5)$ & $121.2(2)$ \\
\hline$C(2)$ & $C(7)$ & $\mathrm{C}(6)$ & $120.8(2)$ & $\mathrm{C}(9)$ & $\mathrm{C}(8)$ & $\mathrm{C}(14)$ & $120.8(1)$ \\
\hline$C(9)$ & $\mathrm{C}(8)$ & $\mathrm{C}(1)$ & $115.7(1)$ & $\mathrm{C}(14)$ & $\mathrm{C}(8)$ & $\mathrm{C}(1)$ & $123.5(1)$ \\
\hline$C(10)$ & $\mathrm{C}(9)$ & $\mathrm{O}(2)$ & $120.1(1)$ & $C(10)$ & $\mathrm{C}(9)$ & $\mathrm{C}(8)$ & $119.8(1)$ \\
\hline $\mathrm{O}(2)$ & $\mathrm{C}(9)$ & $\mathrm{C}(8)$ & $120.1(1)$ & $C(12)$ & $\mathrm{C}(10)$ & $\mathrm{C}(28)$ & $120.8(1)$ \\
\hline$C(12)$ & $\mathrm{C}(10)$ & $\mathrm{C}(9)$ & $117.1(1)$ & $\mathrm{C}(28)$ & $\mathrm{C}(10)$ & $\mathrm{C}(9)$ & $122.1(1)$ \\
\hline$C(13)$ & $C(12)$ & $\mathrm{C}(10)$ & $123.5(2)$ & $C(14)$ & $\mathrm{C}(13)$ & $\mathrm{C}(21)$ & $121.1(1)$ \\
\hline$C(14)$ & $\mathrm{C}(13)$ & $\mathrm{C}(12)$ & $117.6(1)$ & $\mathrm{C}(21)$ & $\mathrm{C}(13)$ & $\mathrm{C}(12)$ & $121.3(1)$ \\
\hline $\mathrm{C}(8)$ & $C(14)$ & $\mathrm{C}(13)$ & $121.0(1)$ & $C(16)$ & $C(15)$ & $\mathrm{C}(17)$ & $107.3(1)$ \\
\hline$C(16)$ & $\mathrm{C}(15)$ & $\mathrm{C}(4)$ & $112.0(2)$ & $\mathrm{C}(17)$ & $\mathrm{C}(15)$ & $\mathrm{C}(4)$ & $109.7(1)$ \\
\hline C(19) & $\mathrm{C}(18)$ & $\mathrm{C}(20)$ & $109.8(2)$ & $C(19)$ & $\mathrm{C}(18)$ & $\mathrm{C}(6)$ & $112.2(2)$ \\
\hline $\mathrm{C}(20)$ & $\mathrm{C}(18)$ & $\mathrm{C}(6)$ & $108.2(2)$ & $\mathrm{C}(22)$ & $\mathrm{C}(21)$ & $\mathrm{C}(23)$ & $120.9(4)$ \\
\hline$C(22)$ & $\mathrm{C}(21)$ & $\mathrm{C}(24)$ & $110.4(3)$ & $\mathrm{C}(22)$ & $\mathrm{C}(21)$ & $\mathrm{C}(25)$ & $18.1(3)$ \\
\hline$C(22)$ & $\mathrm{C}(21)$ & $\mathrm{C}(26)$ & $87.9(3)$ & $\mathrm{C}(22)$ & $\mathrm{C}(21)$ & $\mathrm{C}(27)$ & $117.5(4)$ \\
\hline $\mathrm{C}(22)$ & $\mathrm{C}(21)$ & $\mathrm{C}(13)$ & $111.5(3)$ & $\mathrm{C}(23)$ & $\mathrm{C}(21)$ & $\mathrm{C}(24)$ & $100.3(3)$ \\
\hline$C(23)$ & $\mathrm{C}(21)$ & $\mathrm{C}(25)$ & $137.3(3)$ & $\mathrm{C}(23)$ & $\mathrm{C}(21)$ & $C(26)$ & $35.0(3)$ \\
\hline $\mathrm{C}(23)$ & $\mathrm{C}(21)$ & $\mathrm{C}(27)$ & $84.3(3)$ & $C(23)$ & $\mathrm{C}(21)$ & $\mathrm{C}(13)$ & $106.0(2)$ \\
\hline $\mathrm{C}(24)$ & $\mathrm{C}(21)$ & $\mathrm{C}(25)$ & $96.4(3)$ & $C(24)$ & $\mathrm{C}(21)$ & $C(26)$ & $126.6(3)$ \\
\hline$C(24)$ & $\mathrm{C}(21)$ & $\mathrm{C}(27)$ & $16.2(3)$ & $C(24)$ & $\mathrm{C}(21)$ & $\mathrm{C}(13)$ & $106.4(2)$ \\
\hline$C(25)$ & $\mathrm{C}(21)$ & $\mathrm{C}(26)$ & $105.7(2)$ & $C(25)$ & $\mathrm{C}(21)$ & $\mathrm{C}(27)$ & $106.8(3)$ \\
\hline$C(25)$ & $\mathrm{C}(21)$ & $\mathrm{C}(13)$ & $106.5(2)$ & $C(26)$ & $\mathrm{C}(21)$ & $\mathrm{C}(27)$ & $110.9(3)$ \\
\hline$C(26)$ & $\mathrm{C}(21)$ & $\mathrm{C}(13)$ & $112.5(2)$ & $\mathrm{C}(27)$ & $\mathrm{C}(21)$ & $\mathrm{C}(13)$ & $113.9(3)$ \\
\hline $\mathrm{C}(21)$ & $C(22)$ & $\mathrm{C}(25)$ & $100(1)$ & $\mathrm{C}(21)$ & $\mathrm{C}(23)$ & $\mathrm{C}(26)$ & $68.9(4)$ \\
\hline $\mathrm{C}(21)$ & $C(24)$ & $\mathrm{C}(27)$ & $57.0(8)$ & $\mathrm{C}(21)$ & $\mathrm{C}(25)$ & $\mathrm{C}(22)$ & $61.7(9)$ \\
\hline $\mathrm{C}(21)$ & $C(26)$ & $\mathrm{C}(23)$ & $76.1(4)$ & $\mathrm{C}(21)$ & $\mathrm{C}(27)$ & $\mathrm{C}(24)$ & $106.7(9)$ \\
\hline C(29) & $\mathrm{C}(28)$ & $\mathrm{C}(30)$ & $106.1(2)$ & $C(29)$ & $\mathrm{C}(28)$ & $\mathrm{C}(31)$ & $109.6(2)$ \\
\hline C(29) & $\mathrm{C}(28)$ & $\mathrm{C}(10)$ & $110.4(1)$ & $C(30)$ & $\mathrm{C}(28)$ & $\mathrm{C}(31)$ & $108.0(2)$ \\
\hline $\mathrm{C}(30)$ & $\mathrm{C}(28)$ & $\mathrm{C}(10)$ & $112.9(2)$ & $\mathrm{C}(31)$ & $\mathrm{C}(28)$ & $\mathrm{C}(10)$ & $109.7(1)$ \\
\hline $\mathrm{C}(33)$ & $C(32)$ & $\mathrm{N}(1)$ & $112.6(5)$ & $C(35)$ & $\mathrm{C}(34)$ & $\mathrm{N}(1)$ & $117.1(5)$ \\
\hline $\mathrm{Ti}(1)$ & $\mathrm{H}(1)$ & $\mathrm{C}(1)$ & $168(2)$ & & & & \\
\hline
\end{tabular}


Figure S2. Molecular Structure of 3.

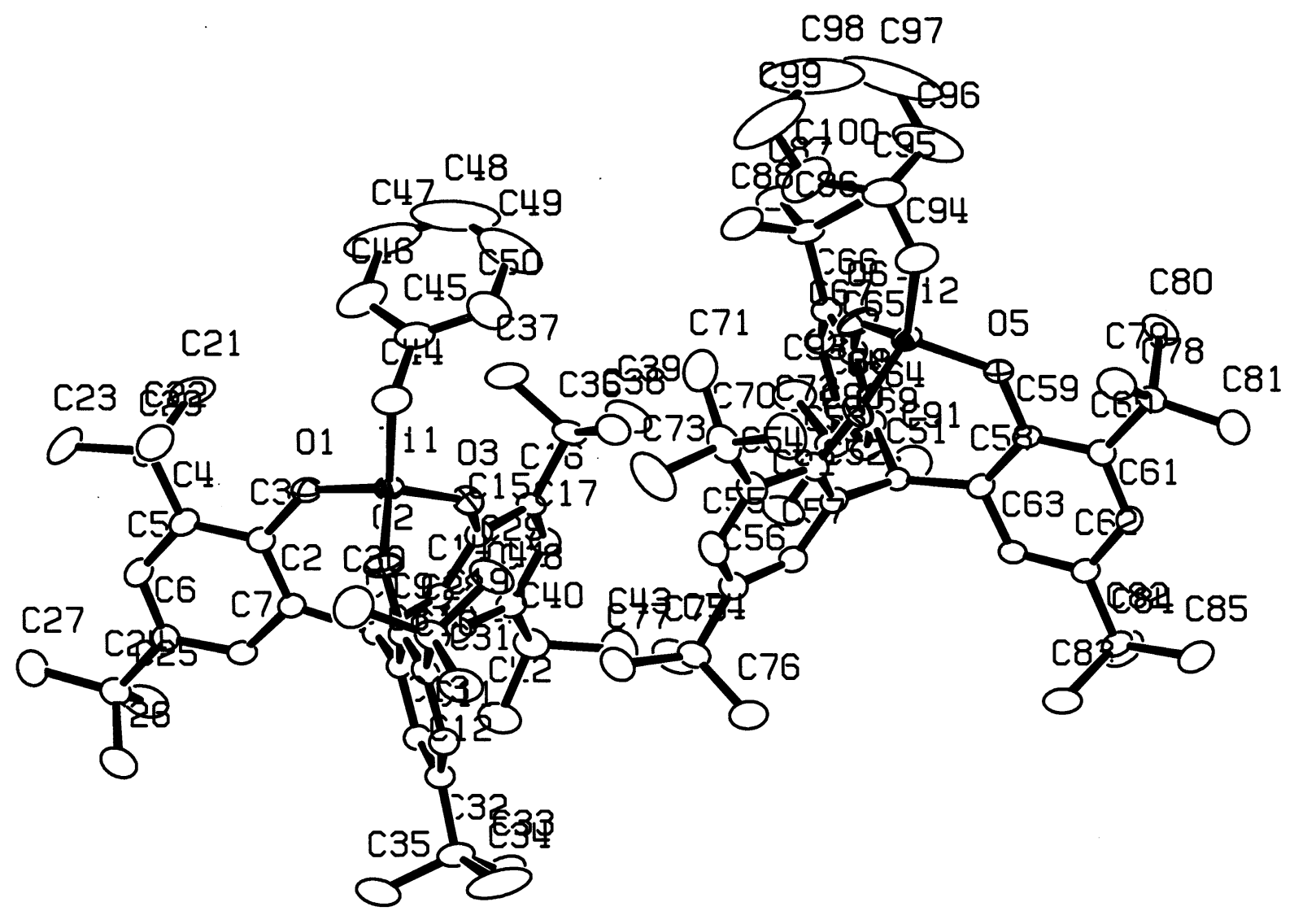


Table S4. Bond Distances ( $\AA$ ) for 3 .

\begin{tabular}{|c|c|c|c|c|c|}
\hline atom & atom & distance & atom & atom & distance \\
\hline $\operatorname{Ti}(1)$ & $\mathrm{O}(1)$ & $1.782(2)$ & $\operatorname{Ti}(1)$ & $\mathrm{O}(2)$ & $1.782(2)$ \\
\hline $\operatorname{Ti}(1)$ & $\mathrm{O}(3)$ & $1.792(2)$ & $\operatorname{Ti}(1)$ & $\mathrm{C}(44)$ & $2.105(3)$ \\
\hline $\operatorname{Ti}(2)$ & $\mathrm{O}(4)$ & $1.784(2)$ & $\operatorname{Ti}(2)$ & $\mathrm{O}(5)$ & $1.784(2)$ \\
\hline $\operatorname{Ti}(2)$ & $\mathrm{O}(6)$ & $1.792(2)$ & $\operatorname{Ti}(2)$ & $\mathrm{C}(94)$ & $2.114(3)$ \\
\hline $\mathrm{O}(1)$ & $\mathrm{C}(3)$ & $1.375(3)$ & $\mathrm{O}(2)$ & $\mathrm{C}(9)$ & $1.366(3)$ \\
\hline $\mathrm{O}(3)$ & $C(15)$ & $1.374(3)$ & $\mathrm{O}(4)$ & $\mathrm{C}(53)$ & $1.369(3)$ \\
\hline $\mathrm{O}(5)$ & C(59) & $1.371(3)$ & $\mathrm{O}(6)$ & $C(65)$ & $1.367(3)$ \\
\hline $\mathrm{C}(1)$ & $\mathrm{C}(2)$ & $1.559(4)$ & $\mathrm{C}(1)$ & $\mathrm{C}(8)$ & $1.557(3)$ \\
\hline $\mathrm{C}(1)$ & $C(14)$ & $1.555(4)$ & $\mathrm{C}(2)$ & $\mathrm{C}(3)$ & $1.406(3)$ \\
\hline $\mathrm{C}(2)$ & $\mathrm{C}(7)$ & $1.398(4)$ & $\mathrm{C}(3)$ & $C(4)$ & $1.404(4)$ \\
\hline$C(4)$ & $C(5)$ & $1.393(4)$ & $C(4)$ & $\mathrm{C}(20)$ & $1.541(4)$ \\
\hline$C(5)$ & $C(6)$ & $1.383(4)$ & $C(6)$ & $C(7)$ & $1.394(4)$ \\
\hline$C(6)$ & $C(24)$ & $1.533(4)$ & $\mathrm{C}(8)$ & $\mathrm{C}(9)$ & $1.408(4)$ \\
\hline $\mathrm{C}(8)$ & $C(13)$ & $1.398(3)$ & $\mathrm{C}(9)$ & $\mathrm{C}(10)$ & $1.415(3)$ \\
\hline$C(10)$ & $\mathrm{C}(11)$ & $1.393(4)$ & $C(10)$ & $\mathrm{C}(28)$ & $1.538(4)$ \\
\hline $\mathrm{C}(11)$ & $C(12)$ & $1.385(4)$ & $\mathrm{C}(12)$ & $\mathrm{C}(13)$ & $1.393(4)$ \\
\hline$C(12)$ & $C(32)$ & $1.540(4)$ & $C(14)$ & $\mathrm{C}(15)$ & $1.405(3)$ \\
\hline$C(14)$ & $C(19)$ & $1.400(4)$ & $C(15)$ & $C(16)$ & $1.415(4)$ \\
\hline$C(16)$ & $C(17)$ & $1.392(4)$ & $C(16)$ & $\mathrm{C}(36)$ & $1.550(4)$ \\
\hline$C(17)$ & $\mathrm{C}(18)$ & $1.392(4)$ & $\mathrm{C}(18)$ & $\mathrm{C}(19)$ & $1.391(4)$ \\
\hline $\mathrm{C}(18)$ & $C(40)$ & $1.536(4)$ & $\mathrm{C}(20)$ & $\mathrm{C}(21)$ & $1.548(5)$ \\
\hline$C(20)$ & $C(22)$ & $1.534(5)$ & $\mathrm{C}(20)$ & $\mathrm{C}(23)$ & $1.533(6)$ \\
\hline$C(24)$ & $C(25)$ & $1.544(5)$ & $C(24)$ & $C(26)$ & $1.544(4)$ \\
\hline$C(24)$ & $C(27)$ & $1.518(5)$ & $\mathrm{C}(28)$ & $\mathrm{C}(29)$ & $1.541(4)$ \\
\hline$C(28)$ & $C(30)$ & $1.526(5)$ & $\mathrm{C}(28)$ & $\mathrm{C}(31)$ & $1.540(4)$ \\
\hline$C(32)$ & $\mathrm{C}(33)$ & $1.527(5)$ & $C(32)$ & $\mathrm{C}(34)$ & $1.523(5)$ \\
\hline$C(32)$ & $C(35)$ & $1.534(5)$ & $C(36)$ & $\mathrm{C}(37)$ & $1.539(4)$ \\
\hline$C(36)$ & $\mathrm{C}(38)$ & $1.537(5)$ & $C(36)$ & $\mathrm{C}(39)$ & $1.534(5)$ \\
\hline$C(40)$ & $\mathrm{C}(41)$ & $1.530(4)$ & $\mathrm{C}(40)$ & $\mathrm{C}(42)$ & $1.531(4)$ \\
\hline$C(40)$ & $C(43)$ & $1.540(5)$ & $C(44)$ & $C(45)$ & $1.488(4)$ \\
\hline$C(45)$ & $C(46)$ & $1.392(5)$ & $C(45)$ & $\mathrm{C}(50)$ & $1.387(5)$ \\
\hline$C(46)$ & $C(47)$ & $1.376(7)$ & $C(47)$ & $\mathrm{C}(48)$ & $1.36(1)$ \\
\hline$C(48)$ & C(49) & $1.390(9)$ & $\mathrm{C}(49)$ & $\mathrm{C}(50)$ & $1.395(6)$ \\
\hline$C(51)$ & $C(52)$ & $1.544(4)$ & $\mathrm{C}(51)$ & $\mathrm{C}(58)$ & $1.555(4)$ \\
\hline$C(51)$ & $C(64)$ & $1.563(3)$ & $\mathrm{C}(52)$ & $\mathrm{C}(53)$ & $1.408(4)$ \\
\hline$C(52)$ & $C(57)$ & $1.382(4)$ & $\mathrm{C}(53)$ & $\mathrm{C}(54)$ & $1.409(4)$ \\
\hline$C(54)$ & $C(55)$ & $1.402(4)$ & $C(54)$ & $\mathrm{C}(70)$ & $1.537(5)$ \\
\hline
\end{tabular}


Table S4. Bond Distances (Å) for 3. (Cont.)

\begin{tabular}{|c|c|c|c|c|c|}
\hline atom & atom & distance & atom & atom & distance \\
\hline$C(55)$ & $C(56)$ & $1.387(4)$ & $C(56)$ & $C(57)$ & $1.402(4)$ \\
\hline$C(56)$ & $\mathrm{C}(74)$ & $1.535(4)$ & $\mathrm{C}(58)$ & $\mathrm{C}(59)$ & $1.407(4)$ \\
\hline $\mathrm{C}(58)$ & $\mathrm{C}(63)$ & $1.394(3)$ & $\mathrm{C}(59)$ & $\mathrm{C}(60)$ & $1.405(4)$ \\
\hline$C(60)$ & $\mathrm{C}(61)$ & $1.396(3)$ & $\mathrm{C}(60)$ & $\mathrm{C}(78)$ & $1.548(4)$ \\
\hline $\mathrm{C}(61)$ & $\mathrm{C}(62)$ & $1.399(4)$ & $\mathrm{C}(62)$ & $\mathrm{C}(63)$ & $1.382(4)$ \\
\hline$C(62)$ & $\mathrm{C}(82)$ & $1.537(4)$ & C(64) & $\mathrm{C}(65)$ & $1.415(3)$ \\
\hline$C(64)$ & $\mathrm{C}(69)$ & $1.396(4)$ & C(65) & $C(66)$ & $1.412(3)$ \\
\hline$C(66)$ & $\mathrm{C}(67)$ & $1.394(4)$ & C(66) & $C(86)$ & $1.544(4)$ \\
\hline$C(67)$ & $\mathrm{C}(68)$ & $1.382(3)$ & C(68) & $\mathrm{C}(69)$ & $1.394(3)$ \\
\hline$C(68)$ & $\mathrm{C}(90)$ & $1.543(4)$ & $\mathrm{C}(70)$ & $\mathrm{C}(71)$ & $1.538(5)$ \\
\hline$C(70)$ & $\mathrm{C}(72)$ & $1.541(5)$ & $\mathrm{C}(70)$ & $\mathrm{C}(73)$ & $1.535(5)$ \\
\hline$C(74)$ & $C(75)$ & $1.533(5)$ & $\mathrm{C}(74)$ & $\mathrm{C}(76)$ & $1.531(5)$ \\
\hline $\mathrm{C}(74)$ & $\mathrm{C}(77)$ & $1.539(5)$ & $\mathrm{C}(78)$ & $\mathrm{C}(79)$ & $1.542(4)$ \\
\hline $\mathrm{C}(78)$ & $\mathrm{C}(80)$ & $1.545(4)$ & $\mathrm{C}(78)$ & $\mathrm{C}(81)$ & $1.532(5)$ \\
\hline $\mathrm{C}(82)$ & $\mathrm{C}(83)$ & $1.536(4)$ & $\mathrm{C}(82)$ & $\mathrm{C}(84)$ & $1.532(5)$ \\
\hline $\mathrm{C}(82)$ & $\mathrm{C}(85)$ & $1.524(5)$ & C(86) & $\mathrm{C}(87)$ & $1.537(4)$ \\
\hline$C(86)$ & $\mathrm{C}(88)$ & $1.535(5)$ & $\mathrm{C}(86)$ & C(89) & $1.533(5)$ \\
\hline $\mathrm{C}(90)$ & $\mathrm{C}(91)$ & $1.527(5)$ & $\mathrm{C}(90)$ & $\mathrm{C}(92)$ & $1.538(4)$ \\
\hline $\mathrm{C}(90)$ & $\mathrm{C}(93)$ & $1.530(4)$ & C(94) & $\mathrm{C}(95)$ & $1.469(4)$ \\
\hline$C(95)$ & $\mathrm{C}(96)$ & $1.381(6)$ & C(95) & $\mathrm{C}(100)$ & $1.390(7)$ \\
\hline $\mathrm{C}(96)$ & $\mathrm{C}(97)$ & $1.48(1)$ & C(97) & $\mathrm{C}(98)$ & $1.40(2)$ \\
\hline $\mathrm{C}(98)$ & C(99) & $1.32(2)$ & C(99) & $\mathrm{C}(100)$ & $1.337(8)$ \\
\hline $\mathrm{C}(1)$ & $\mathrm{H}(1)$ & $0.94(3)$ & $\mathrm{C}(51)$ & $\mathrm{H}(69)$ & $0.98(3)$ \\
\hline
\end{tabular}


Table S5. Bond Angles (deg) for 3.

\begin{tabular}{|c|c|c|c|c|c|c|c|}
\hline atom & atom & atom & angle & atom & atom & atom & angle \\
\hline $\mathrm{O}(1)$ & $\operatorname{Ti}(1)$ & $\mathrm{O}(2)$ & $104.6(1)$ & $\mathrm{O}(1)$ & $\operatorname{Ti}(1)$ & $\mathrm{O}(3)$ & $104.0(1)$ \\
\hline $\mathrm{O}(1)$ & $\operatorname{Ti}(1)$ & $\mathrm{C}(44)$ & $113.4(1)$ & $\mathrm{O}(2)$ & $\operatorname{Ti}(1)$ & $\mathrm{O}(3)$ & $106.23(9)$ \\
\hline $\mathrm{O}(2)$ & $\operatorname{Ti}(1)$ & $\mathrm{C}(44)$ & 109.7(1) & $\mathrm{O}(3)$ & $\operatorname{Ti}(1)$ & $\mathrm{C}(44)$ & $117.9(1)$ \\
\hline $\mathrm{O}(4)$ & $\operatorname{Ti}(2)$ & $\mathrm{O}(5)$ & $105.44(9)$ & $\mathrm{O}(4)$ & $\operatorname{Ti}(2)$ & $\mathrm{O}(6)$ & 104.3(1) \\
\hline $\mathrm{O}(4)$ & $\operatorname{Ti}(2)$ & $\mathrm{C}(94)$ & $109.8(1)$ & $\mathrm{O}(5)$ & $\mathrm{Ti}(2)$ & $\mathrm{O}(6)$ & 104.7(1) \\
\hline $\mathrm{O}(5)$ & $\operatorname{Ti}(2)$ & $\mathrm{C}(94)$ & $111.2(1)$ & $\mathrm{O}(6)$ & $\operatorname{Ti}(2)$ & $\mathrm{C}(94)$ & $120.2(1)$ \\
\hline$C(3)$ & $\mathrm{O}(1)$ & $\operatorname{Ti}(1)$ & $137.2(2)$ & $\mathrm{C}(9)$ & $\mathrm{O}(2)$ & $\operatorname{Ti}(1)$ & $137.7(2)$ \\
\hline$C(15)$ & $\mathrm{O}(3)$ & $\operatorname{Ti}(1)$ & $135.8(2)$ & $\mathrm{C}(53)$ & $\mathrm{O}(4)$ & $\operatorname{Ti}(2)$ & $138.4(2)$ \\
\hline$C(59)$ & $\mathrm{O}(5)$ & $\operatorname{Ti}(2)$ & $133.6(2)$ & $C(65)$ & $\mathrm{O}(6)$ & $\operatorname{Ti}(2)$ & $137.6(2)$ \\
\hline $\mathrm{C}(2)$ & $\mathrm{C}(1)$ & $\mathrm{C}(8)$ & $118.7(2)$ & $\mathrm{C}(2)$ & $\mathrm{C}(1)$ & $\mathrm{C}(14)$ & $116.7(2)$ \\
\hline $\mathrm{C}(8)$ & $\mathrm{C}(1)$ & $\mathrm{C}(14)$ & $118.0(2)$ & $\mathrm{C}(3)$ & $\mathrm{C}(2)$ & $C(7)$ & $116.5(2)$ \\
\hline$C(3)$ & $\mathrm{C}(2)$ & $\mathrm{C}(1)$ & $128.7(2)$ & $C(7)$ & $C(2)$ & $\mathrm{C}(1)$ & $114.8(2)$ \\
\hline$C(4)$ & $\mathrm{C}(3)$ & $\mathrm{O}(1)$ & $117.6(2)$ & $\mathrm{C}(4)$ & $\mathrm{C}(3)$ & $\mathrm{C}(2)$ & $122.2(2)$ \\
\hline $\mathrm{O}(1)$ & $\mathrm{C}(3)$ & $\mathrm{C}(2)$ & $120.2(2)$ & $C(5)$ & $\mathrm{C}(4)$ & $\mathrm{C}(20)$ & $120.9(3)$ \\
\hline$C(5)$ & $\mathrm{C}(4)$ & $\mathrm{C}(3)$ & $117.3(2)$ & $\mathrm{C}(20)$ & $\mathrm{C}(4)$ & $\mathrm{C}(3)$ & $121.8(3)$ \\
\hline$C(6)$ & $C(5)$ & $\mathrm{C}(4)$ & $123.6(3)$ & $\mathrm{C}(7)$ & $\mathrm{C}(6)$ & $\mathrm{C}(24)$ & $120.5(2)$ \\
\hline$C(7)$ & $C(6)$ & $C(5)$ & $116.6(3)$ & $\mathrm{C}(24)$ & $\mathrm{C}(6)$ & $\mathrm{C}(5)$ & $122.9(3)$ \\
\hline$C(2)$ & $C(7)$ & $\mathrm{C}(6)$ & $123.9(2)$ & $\mathrm{C}(9)$ & $\mathrm{C}(8)$ & $\mathrm{C}(13)$ & $116.6(2)$ \\
\hline$C(9)$ & $\mathrm{C}(8)$ & $\mathrm{C}(1)$ & $128.6(2)$ & $\mathrm{C}(13)$ & $\mathrm{C}(8)$ & $\mathrm{C}(1)$ & $114.7(2)$ \\
\hline$C(10)$ & $\mathrm{C}(9)$ & $\mathrm{O}(2)$ & $118.6(2)$ & $C(10)$ & $\mathrm{C}(9)$ & $\mathrm{C}(8)$ & $122.2(2)$ \\
\hline $\mathrm{O}(2)$ & $\mathrm{C}(9)$ & $\mathrm{C}(8)$ & $119.2(2)$ & $\mathrm{C}(11)$ & $C(10)$ & $\mathrm{C}(28)$ & $121.1(2)$ \\
\hline$C(11)$ & $\mathrm{C}(10)$ & $\mathrm{C}(9)$ & $117.1(2)$ & $\mathrm{C}(28)$ & $C(10)$ & $\mathrm{C}(9)$ & $121.8(2)$ \\
\hline$C(12)$ & $\mathrm{C}(11)$ & $\mathrm{C}(10)$ & $123.2(2)$ & $\mathrm{C}(13)$ & $C(12)$ & $C(32)$ & $120.3(3)$ \\
\hline$C(13)$ & $C(12)$ & $\mathrm{C}(11)$ & $117.4(2)$ & $C(32)$ & $C(12)$ & $\mathrm{C}(11)$ & $122.2(2)$ \\
\hline $\mathrm{C}(8)$ & $C(13)$ & $\mathrm{C}(12)$ & $123.4(3)$ & $C(15)$ & $C(14)$ & $C(19)$ & $116.6(2)$ \\
\hline$C(15)$ & $C(14)$ & $\mathrm{C}(1)$ & $129.4(2)$ & $\mathrm{C}(19)$ & $C(14)$ & $\mathrm{C}(1)$ & $114.0(2)$ \\
\hline$C(16)$ & $C(15)$ & $\mathrm{O}(3)$ & $117.8(2)$ & $C(16)$ & $C(15)$ & $C(14)$ & $122.2(2)$ \\
\hline $\mathrm{O}(3)$ & $C(15)$ & $\mathrm{C}(14)$ & $120.0(2)$ & $\mathrm{C}(17)$ & $C(16)$ & $C(36)$ & $120.2(2)$ \\
\hline$C(17)$ & $C(16)$ & $C(15)$ & $117.1(2)$ & $C(36)$ & $C(16)$ & $C(15)$ & $122.7(2)$ \\
\hline $\mathrm{C}(18)$ & $\mathrm{C}(17)$ & $C(16)$ & $123.3(2)$ & $\mathrm{C}(19)$ & $\mathrm{C}(18)$ & $C(40)$ & $122.9(2)$ \\
\hline$C(19)$ & $\mathrm{C}(18)$ & $\mathrm{C}(17)$ & $116.9(2)$ & $\mathrm{C}(40)$ & $\mathrm{C}(18)$ & $\mathrm{C}(17)$ & $120.2(2)$ \\
\hline$C(14)$ & $C(19)$ & $\mathrm{C}(18)$ & $123.6(2)$ & $\mathrm{C}(21)$ & $\mathrm{C}(20)$ & $C(22)$ & $110.5(3)$ \\
\hline $\mathrm{C}(21)$ & $\mathrm{C}(20)$ & $\mathrm{C}(23)$ & $107.6(3)$ & $\mathrm{C}(21)$ & $C(20)$ & $\mathrm{C}(4)$ & $110.6(3)$ \\
\hline$C(22)$ & $\mathrm{C}(20)$ & $\mathrm{C}(23)$ & $106.9(3)$ & $\mathrm{C}(22)$ & $C(20)$ & $C(4)$ & $109.8(2)$ \\
\hline$C(23)$ & $\mathrm{C}(20)$ & $\mathrm{C}(4)$ & $111.4(3)$ & $C(25)$ & $C(24)$ & $C(26)$ & $108.8(3)$ \\
\hline$C(25)$ & $\mathrm{C}(24)$ & $\mathrm{C}(27)$ & $108.4(3)$ & $C(25)$ & $\mathrm{C}(24)$ & $C(6)$ & $109.3(2)$ \\
\hline$C(26)$ & $\mathrm{C}(24)$ & $\mathrm{C}(27)$ & 108.1(3) & $C(26)$ & $\mathrm{C}(24)$ & $\mathrm{C}(6)$ & $110.4(2)$ \\
\hline
\end{tabular}


Table S5. Bond Angles (deg) for 3. (Cont.)

\begin{tabular}{|c|c|c|c|c|c|c|c|}
\hline atom & atom & atom & angle & atom & atom & atom & angle \\
\hline $\mathrm{C}(27)$ & $\mathrm{C}(24)$ & $\mathrm{C}(6)$ & $111.8(3)$ & $\mathrm{C}(29)$ & $\mathrm{C}(28)$ & $\mathrm{C}(30)$ & $111.0(3)$ \\
\hline$C(29)$ & $\mathrm{C}(28)$ & $\mathrm{C}(31)$ & $105.7(3)$ & $C(29)$ & $\mathrm{C}(28)$ & $C(10)$ & $109.8(2)$ \\
\hline $\mathrm{C}(30)$ & $\mathrm{C}(28)$ & $\mathrm{C}(31)$ & $107.6(3)$ & $C(30)$ & $\mathrm{C}(28)$ & $C(10)$ & $110.3(3)$ \\
\hline $\mathrm{C}(31)$ & $\mathrm{C}(28)$ & $\mathrm{C}(10)$ & $112.4(2)$ & $C(33)$ & $\mathrm{C}(32)$ & $\mathrm{C}(34)$ & $108.2(3)$ \\
\hline $\mathrm{C}(33)$ & $\mathrm{C}(32)$ & $\mathrm{C}(35)$ & $109.6(3)$ & $C(33)$ & $\mathrm{C}(32)$ & $\mathrm{C}(12)$ & $111.3(2)$ \\
\hline $\mathrm{C}(34)$ & $\mathrm{C}(32)$ & $\mathrm{C}(35)$ & $107.7(3)$ & $C(34)$ & $\mathrm{C}(32)$ & $\mathrm{C}(12)$ & $111.9(3)$ \\
\hline$C(35)$ & $C(32)$ & $\mathrm{C}(12)$ & $108.0(3)$ & $C(37)$ & $\mathrm{C}(36)$ & $\mathrm{C}(38)$ & $110.4(2)$ \\
\hline $\mathrm{C}(37)$ & $C(36)$ & $\mathrm{C}(39)$ & $108.4(2)$ & $C(37)$ & $C(36)$ & $C(16)$ & $108.3(2)$ \\
\hline $\mathrm{C}(38)$ & $\mathrm{C}(36)$ & $\mathrm{C}(39)$ & 106.2(3) & $\mathrm{C}(38)$ & $\mathrm{C}(36)$ & $\mathrm{C}(16)$ & $112.2(2)$ \\
\hline $\mathrm{C}(39)$ & $C(36)$ & $\mathrm{C}(16)$ & $111.3(2)$ & $C(41)$ & $\mathrm{C}(40)$ & $\mathrm{C}(42)$ & $108.4(3)$ \\
\hline $\mathrm{C}(41)$ & $\mathrm{C}(40)$ & $\mathrm{C}(43)$ & $109.7(3)$ & $C(41)$ & $\mathrm{C}(40)$ & $C(18)$ & $108.6(2)$ \\
\hline $\mathrm{C}(42)$ & $\mathrm{C}(40)$ & $\mathrm{C}(43)$ & 107.4(3) & $\mathrm{C}(42)$ & $\mathrm{C}(40)$ & $\mathrm{C}(18)$ & $112.3(2)$ \\
\hline $\mathrm{C}(43)$ & $\mathrm{C}(40)$ & $\mathrm{C}(18)$ & $110.3(3)$ & $C(45)$ & $\mathrm{C}(44)$ & $\mathrm{Ti}(1)$ & $111.8(2)$ \\
\hline $\mathrm{C}(46)$ & $C(45)$ & $\mathrm{C}(50)$ & $117.5(3)$ & $C(46)$ & $\mathrm{C}(45)$ & $\mathrm{C}(44)$ & 121.1(3) \\
\hline $\mathrm{C}(50)$ & $C(45)$ & $\mathrm{C}(44)$ & $121.4(3)$ & $\mathrm{C}(47)$ & $\mathrm{C}(46)$ & $\mathrm{C}(45)$ & $121.5(5)$ \\
\hline $\mathrm{C}(48)$ & $\mathrm{C}(47)$ & $C(46)$ & $120.8(5)$ & $C(49)$ & $\mathrm{C}(48)$ & $\mathrm{C}(47)$ & $119.4(6)$ \\
\hline $\mathrm{C}(50)$ & $\mathrm{C}(49)$ & $\mathrm{C}(48)$ & $120.0(6)$ & $\mathrm{C}(45)$ & $C(50)$ & $\mathrm{C}(49)$ & $120.7(4)$ \\
\hline $\mathrm{C}(52)$ & $\mathrm{C}(51)$ & $\mathrm{C}(58)$ & $115.5(2)$ & $\mathrm{C}(52)$ & $\mathrm{C}(51)$ & $\mathrm{C}(64)$ & $115.4(2)$ \\
\hline $\mathrm{C}(58)$ & $\mathrm{C}(51)$ & $\mathrm{C}(64)$ & $122.4(2)$ & $C(53)$ & $C(52)$ & $\mathrm{C}(57)$ & $117.1(2)$ \\
\hline $\mathrm{C}(53)$ & $C(52)$ & $\mathrm{C}(51)$ & $126.8(2)$ & C (57) & $\mathrm{C}(52)$ & $\mathrm{C}(51)$ & $116.1(2)$ \\
\hline $\mathrm{C}(54)$ & $\mathrm{C}(53)$ & $\mathrm{O}(4)$ & $118.7(2)$ & $C(54)$ & $\mathrm{C}(53)$ & $\mathrm{C}(52)$ & $122.1(3)$ \\
\hline $\mathrm{O}(4)$ & $C(53)$ & $\mathrm{C}(52)$ & $119.2(2)$ & $C(55)$ & $\mathrm{C}(54)$ & $\mathrm{C}(70)$ & $121.5(3)$ \\
\hline $\mathrm{C}(55)$ & $\mathrm{C}(54)$ & $\mathrm{C}(53)$ & 117.1(3) & $C(70)$ & $\mathrm{C}(54)$ & $\mathrm{C}(53)$ & $121.2(2)$ \\
\hline $\mathrm{C}(56)$ & $C(55)$ & $\mathrm{C}(54)$ & $122.8(3)$ & $C(57)$ & $C(56)$ & $C(74)$ & $120.2(3)$ \\
\hline C(57) & $\mathrm{C}(56)$ & $\mathrm{C}(55)$ & $117.2(2)$ & $\mathrm{C}(74)$ & $\mathrm{C}(56)$ & $\mathrm{C}(55)$ & $122.5(3)$ \\
\hline $\mathrm{C}(52)$ & $\mathrm{C}(57)$ & $\mathrm{C}(56)$ & $123.5(3)$ & $\mathrm{C}(59)$ & $\mathrm{C}(58)$ & $C(63)$ & $116.9(2)$ \\
\hline $\mathrm{C}(59)$ & $\mathrm{C}(58)$ & $\mathrm{C}(51)$ & $129.8(2)$ & $C(63)$ & $\mathrm{C}(58)$ & $\mathrm{C}(51)$ & $113.3(2)$ \\
\hline $\mathrm{C}(60)$ & C(59) & $\mathrm{O}(5)$ & $118.3(2)$ & $\mathrm{C}(60)$ & $\mathrm{C}(59)$ & $\mathrm{C}(58)$ & $122.1(2)$ \\
\hline $\mathrm{O}(5)$ & $\mathrm{C}(59)$ & $\mathrm{C}(58)$ & $119.5(2)$ & $C(61)$ & $\mathrm{C}(60)$ & $\mathrm{C}(78)$ & $120.2(2)$ \\
\hline $\mathrm{C}(61)$ & $\mathrm{C}(60)$ & $\mathrm{C}(59)$ & $117.5(2)$ & $C(78)$ & $\mathrm{C}(60)$ & $\mathrm{C}(59)$ & $122.3(2)$ \\
\hline $\mathrm{C}(62)$ & $\mathrm{C}(61)$ & $\mathrm{C}(60)$ & $122.5(2)$ & $C(63)$ & $\mathrm{C}(62)$ & $\mathrm{C}(82)$ & $119.6(2)$ \\
\hline $\mathrm{C}(63)$ & $C(62)$ & $\mathrm{C}(61)$ & $117.4(2)$ & $C(82)$ & $C(62)$ & $\mathrm{C}(61)$ & $122.9(2)$ \\
\hline $\mathrm{C}(58)$ & $C(63)$ & $\mathrm{C}(62)$ & $123.6(2)$ & $C(65)$ & $\mathrm{C}(64)$ & $\mathrm{C}(69)$ & $116.4(2)$ \\
\hline $\mathrm{C}(65)$ & $C(64)$ & $\mathrm{C}(51)$ & $129.4(2)$ & $C(69)$ & $\mathrm{C}(64)$ & $\mathrm{C}(51)$ & $113.9(2)$ \\
\hline $\mathrm{C}(66)$ & $C(65)$ & $\mathrm{O}(6)$ & 118.2(2) & $C(66)$ & $C(65)$ & $C(64)$ & $121.4(2)$ \\
\hline$O(6)$ & $\mathrm{C}(65)$ & $\mathrm{C}(64)$ & $120.4(2)$ & $\mathrm{C}(67)$ & $\mathrm{C}(66)$ & $\mathrm{C}(86)$ & $120.9(2)$ \\
\hline $\mathrm{C}(67)$ & $C(66)$ & $\mathrm{C}(65)$ & $118.0(2)$ & $C(86)$ & $C(66)$ & $C(65)$ & $121.1(2)$ \\
\hline
\end{tabular}


Table S5. Bond Angles (deg) for 3. (Cont.)

\begin{tabular}{|c|c|c|c|c|c|c|c|}
\hline atom & atom & atom & angle & atom & atom & atom & angle \\
\hline$C(68)$ & $\mathrm{C}(67)$ & $C(66)$ & $123.1(2)$ & $\mathrm{C}(69)$ & $C(68)$ & $\mathrm{C}(90)$ & $119.9(2)$ \\
\hline C(69) & $\mathrm{C}(68)$ & $\mathrm{C}(67)$ & $116.8(2)$ & $\mathrm{C}(90)$ & $\mathrm{C}(68)$ & $\mathrm{C}(67)$ & $123.4(2)$ \\
\hline$C(64)$ & C(69) & $\mathrm{C}(68)$ & $124.3(2)$ & $\mathrm{C}(71)$ & $C(70)$ & $C(72)$ & 109.1(3) \\
\hline $\mathrm{C}(71)$ & $\mathrm{C}(70)$ & $\mathrm{C}(73)$ & $107.6(3)$ & $\mathrm{C}(71)$ & $C(70)$ & $\mathrm{C}(54)$ & $109.6(3)$ \\
\hline $\mathrm{C}(72)$ & $\mathrm{C}(70)$ & $\mathrm{C}(73)$ & $107.2(3)$ & $\mathrm{C}(72)$ & $C(70)$ & $\mathrm{C}(54)$ & $111.9(3)$ \\
\hline $\mathrm{C}(73)$ & $\mathrm{C}(70)$ & $\mathrm{C}(54)$ & $111.4(3)$ & $\mathrm{C}(75)$ & $C(74)$ & $C(76)$ & $109.5(3)$ \\
\hline$C(75)$ & $\mathrm{C}(74)$ & $\mathrm{C}(77)$ & $106.9(3)$ & $C(75)$ & $C(74)$ & $C(56)$ & $111.4(2)$ \\
\hline$C(76)$ & $\mathrm{C}(74)$ & $\mathrm{C}(77)$ & $109.2(3)$ & $C(76)$ & $\mathrm{C}(74)$ & $C(56)$ & 108.1(3) \\
\hline $\mathrm{C}(77)$ & $\mathrm{C}(74)$ & $C(56)$ & $111.7(3)$ & $\mathrm{C}(79)$ & $\mathrm{C}(78)$ & $\mathrm{C}(80)$ & $109.3(2)$ \\
\hline $\mathrm{C}(79)$ & $\mathrm{C}(78)$ & $\mathrm{C}(81)$ & $107.3(3)$ & $\mathrm{C}(79)$ & $\mathrm{C}(78)$ & $C(60)$ & $109.4(2)$ \\
\hline$C(80)$ & $\mathrm{C}(78)$ & $\mathrm{C}(81)$ & $106.6(2)$ & $\mathrm{C}(80)$ & $\mathrm{C}(78)$ & $\mathrm{C}(60)$ & $112.3(2)$ \\
\hline $\mathrm{C}(81)$ & $\mathrm{C}(78)$ & $\mathrm{C}(60)$ & $111.7(2)$ & $\mathrm{C}(83)$ & $C(82)$ & $\mathrm{C}(84)$ & $108.6(3)$ \\
\hline $\mathrm{C}(83)$ & $\mathrm{C}(82)$ & $\mathrm{C}(85)$ & $108.7(2)$ & $\mathrm{C}(83)$ & $\mathrm{C}(82)$ & $C(62)$ & $110.7(2)$ \\
\hline$C(84)$ & $\mathrm{C}(82)$ & $C(85)$ & $109.0(3)$ & $\mathrm{C}(84)$ & $C(82)$ & $C(62)$ & $107.6(2)$ \\
\hline $\mathrm{C}(85)$ & $\mathrm{C}(82)$ & $C(62)$ & $112.2(2)$ & $\mathrm{C}(87)$ & $C(86)$ & $\mathrm{C}(88)$ & $107.8(3)$ \\
\hline $\mathrm{C}(87)$ & $\mathrm{C}(86)$ & C(89) & $106.3(3)$ & $\mathrm{C}(87)$ & $C(86)$ & $C(66)$ & $111.4(2)$ \\
\hline $\mathrm{C}(88)$ & $\mathrm{C}(86)$ & $\mathrm{C}(89)$ & $111.0(3)$ & $\mathrm{C}(88)$ & $C(86)$ & $C(66)$ & $110.9(2)$ \\
\hline C(89) & $\mathrm{C}(86)$ & $\mathrm{C}(66)$ & $109.3(3)$ & $\mathrm{C}(91)$ & $\mathrm{C}(90)$ & $\mathrm{C}(92)$ & $109.8(2)$ \\
\hline $\mathrm{C}(91)$ & $\mathrm{C}(90)$ & $\mathrm{C}(93)$ & $108.2(2)$ & $\mathrm{C}(91)$ & $\mathrm{C}(90)$ & $C(68)$ & $108.9(2)$ \\
\hline $\mathrm{C}(92)$ & C(90) & $\mathrm{C}(93)$ & $108.2(3)$ & $\mathrm{C}(92)$ & $\mathrm{C}(90)$ & $\mathrm{C}(68)$ & $109.6(2)$ \\
\hline $\mathrm{C}(93)$ & C(90) & $\mathrm{C}(68)$ & $112.0(2)$ & $\mathrm{C}(95)$ & $\mathrm{C}(94)$ & $\operatorname{Ti}(2)$ & $111.2(2)$ \\
\hline$C(96)$ & C(95) & $\mathrm{C}(100)$ & $118.2(4)$ & $\mathrm{C}(96)$ & $C(95)$ & $\mathrm{C}(94)$ & $122.0(4)$ \\
\hline $\mathrm{C}(100)$ & C(95) & $\mathrm{C}(94)$ & $119.8(3)$ & $\mathrm{C}(97)$ & $C(96)$ & $C(95)$ & $119.7(6)$ \\
\hline $\mathrm{C}(98)$ & C(97) & $C(96)$ & $112.7(7)$ & $\mathrm{C}(99)$ & $\mathrm{C}(98)$ & $\mathrm{C}(97)$ & $128.5(8)$ \\
\hline $\mathrm{C}(100)$ & C(99) & $\mathrm{C}(98)$ & $115.6(9)$ & $\mathrm{C}(95)$ & $\mathrm{C}(100)$ & $\mathrm{C}(99)$ & $125.2(6)$ \\
\hline
\end{tabular}


Figure S3. Molecular Structure of 5.

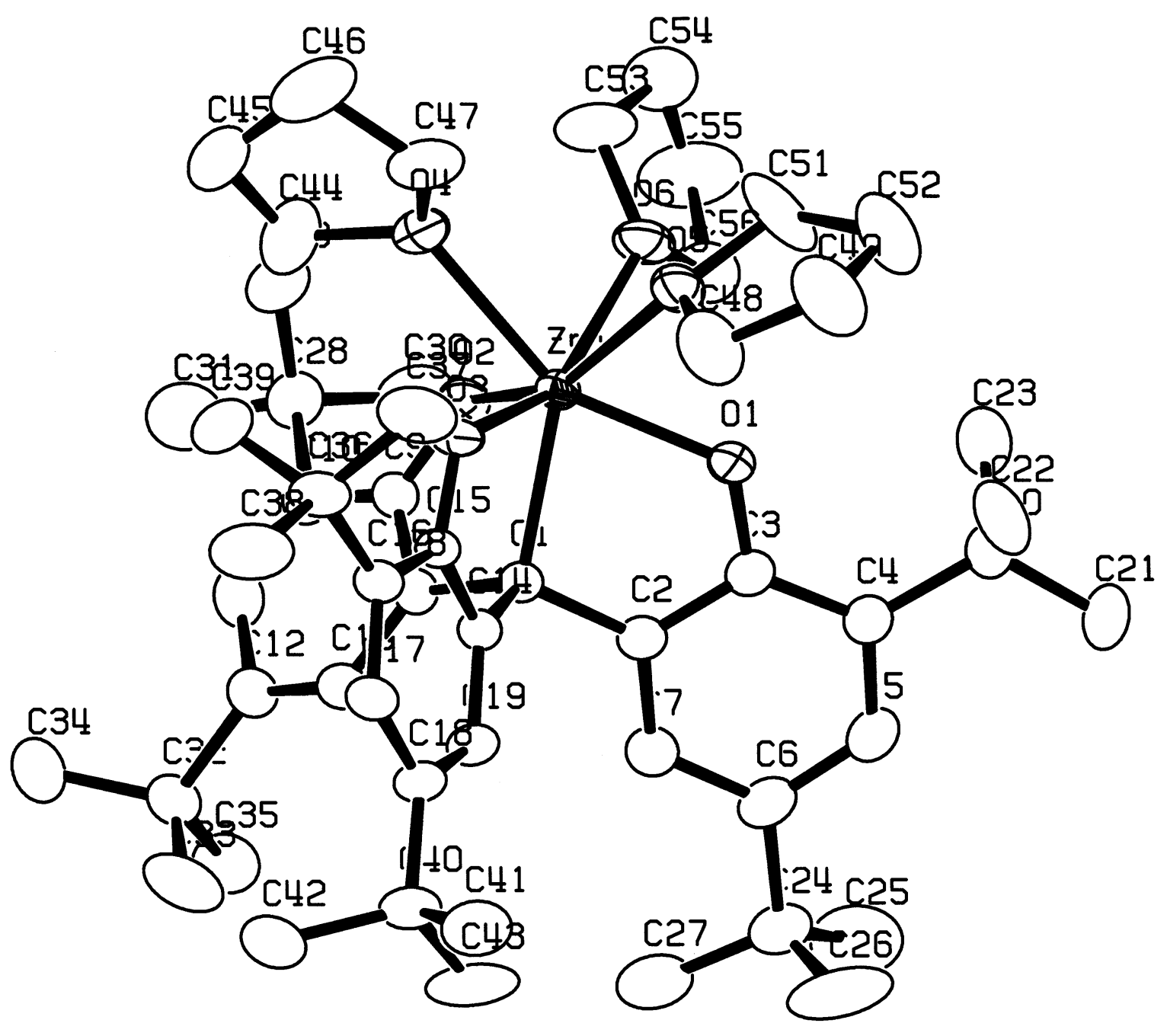


Table S6. Bond Distances ( $\AA$ ) for $\mathbf{5}$.

\begin{tabular}{|c|c|c|c|c|c|}
\hline atom & atom & distance & atom & atom & distance \\
\hline $\operatorname{Zr}(1)$ & $\mathrm{O}(1)$ & $2.049(2)$ & $\operatorname{Zr}(1)$ & $\mathrm{O}(2)$ & $2.036(2)$ \\
\hline $\operatorname{Zr}(1)$ & $\mathrm{O}(3)$ & $2.045(2)$ & $\operatorname{Zr}(1)$ & $\mathrm{O}(4)$ & $2.413(3)$ \\
\hline $\operatorname{Zr}(1)$ & $\mathrm{O}(5)$ & $2.372(3)$ & $\operatorname{Zr}(1)$ & $\mathrm{O}(6)$ & $2.389(3)$ \\
\hline $\operatorname{Zr}(1)$ & $\mathrm{C}(1)$ & $2.309(3)$ & $\mathrm{O}(1)$ & $\mathrm{C}(3)$ & $1.370(4)$ \\
\hline $\mathrm{O}(2)$ & $\mathrm{C}(9)$ & $1.372(4)$ & $\mathrm{O}(3)$ & $\mathrm{C}(15)$ & $1.363(4)$ \\
\hline $\mathrm{O}(4)$ & $\mathrm{C}(44)$ & $1.458(6)$ & $\mathrm{O}(4)$ & $\mathrm{C}(47)$ & $1.449(6)$ \\
\hline $\mathrm{O}(5)$ & $\mathrm{C}(48)$ & $1.474(6)$ & $\mathrm{O}(5)$ & $\mathrm{C}(51)$ & $1.455(6)$ \\
\hline $\mathrm{O}(6)$ & $C(53)$ & $1.455(6)$ & $\mathrm{O}(6)$ & $C(56)$ & $1.457(6)$ \\
\hline $\mathrm{C}(1)$ & $\mathrm{C}(2)$ & $1.520(5)$ & $\mathrm{C}(1)$ & $\mathrm{C}(8)$ & $1.519(5)$ \\
\hline $\mathrm{C}(1)$ & $\mathrm{C}(14)$ & $1.517(5)$ & $\mathrm{C}(2)$ & $\mathrm{C}(3)$ & $1.397(5)$ \\
\hline$C(2)$ & $\mathrm{C}(7)$ & $1.407(5)$ & $\mathrm{C}(3)$ & $\mathrm{C}(4)$ & $1.397(5)$ \\
\hline $\mathrm{C}(4)$ & $C(5)$ & $1.403(6)$ & $\mathrm{C}(4)$ & $\mathrm{C}(20)$ & $1.551(6)$ \\
\hline$C(5)$ & $\mathrm{C}(6)$ & $1.399(6)$ & $C(6)$ & $C(7)$ & $1.385(5)$ \\
\hline$C(6)$ & $\mathrm{C}(24)$ & $1.545(6)$ & $\mathrm{C}(8)$ & $\mathrm{C}(9)$ & $1.410(5)$ \\
\hline $\mathrm{C}(8)$ & $C(13)$ & $1.376(5)$ & $\mathrm{C}(9)$ & $\mathrm{C}(10)$ & $1.410(5)$ \\
\hline$C(10)$ & $\mathrm{C}(11)$ & $1.394(5)$ & $\mathrm{C}(10)$ & $\mathrm{C}(28)$ & $1.534(5)$ \\
\hline $\mathrm{C}(11)$ & $C(12)$ & $1.403(6)$ & $\mathrm{C}(12)$ & $\mathrm{C}(13)$ & $1.382(5)$ \\
\hline $\mathrm{C}(12)$ & $C(32)$ & $1.536(5)$ & $\mathrm{C}(14)$ & $\mathrm{C}(15)$ & $1.397(5)$ \\
\hline$C(14)$ & $\mathrm{C}(19)$ & $1.391(5)$ & $\mathrm{C}(15)$ & $C(16)$ & $1.413(5)$ \\
\hline$C(16)$ & $\mathrm{C}(17)$ & $1.395(5)$ & $C(16)$ & $\mathrm{C}(36)$ & $1.539(5)$ \\
\hline $\mathrm{C}(17)$ & $\mathrm{C}(18)$ & $1.399(5)$ & $\mathrm{C}(18)$ & $\mathrm{C}(19)$ & $1.378(5)$ \\
\hline $\mathrm{C}(18)$ & $\mathrm{C}(40)$ & $1.532(5)$ & $\mathrm{C}(20)$ & $\mathrm{C}(21)$ & $1.532(7)$ \\
\hline $\mathrm{C}(20)$ & $C(22)$ & $1.511(7)$ & $\mathrm{C}(20)$ & $\mathrm{C}(23)$ & $1.540(7)$ \\
\hline $\mathrm{C}(24)$ & $C(25)$ & $1.517(7)$ & $\mathrm{C}(24)$ & $C(26)$ & $1.508(8)$ \\
\hline $\mathrm{C}(24)$ & $\mathrm{C}(27)$ & $1.510(7)$ & $\mathrm{C}(28)$ & $\mathrm{C}(29)$ & $1.525(6)$ \\
\hline $\mathrm{C}(28)$ & $\mathrm{C}(30)$ & $1.538(6)$ & $\mathrm{C}(28)$ & $\mathrm{C}(31)$ & $1.528(7)$ \\
\hline$C(32)$ & $\mathrm{C}(33)$ & $1.539(7)$ & $\mathrm{C}(32)$ & $\mathrm{C}(34)$ & $1.500(7)$ \\
\hline$C(32)$ & $C(35)$ & $1.521(6)$ & $C(36)$ & $\mathrm{C}(37)$ & $1.507(6)$ \\
\hline$C(36)$ & $\mathrm{C}(38)$ & $1.533(6)$ & $C(36)$ & $\mathrm{C}(39)$ & $1.523(6)$ \\
\hline$C(40)$ & $\mathrm{C}(41)$ & $1.544(6)$ & $\mathrm{C}(40)$ & $C(42)$ & $1.525(6)$ \\
\hline$C(40)$ & $C(43)$ & $1.520(7)$ & $\mathrm{C}(44)$ & $\mathrm{C}(45)$ & $1.468(7)$ \\
\hline$C(45)$ & $C(46)$ & $1.498(9)$ & $C(46)$ & $\mathrm{C}(47)$ & $1.484(8)$ \\
\hline $\mathrm{C}(48)$ & $\mathrm{C}(49)$ & $1.495(7)$ & $\mathrm{C}(49)$ & $\mathrm{C}(50)$ & $1.56(2)$ \\
\hline$C(49)$ & $C(52)$ & $1.49(2)$ & $\mathrm{C}(50)$ & $\mathrm{C}(51)$ & $1.46(1)$ \\
\hline$C(50)$ & $C(52)$ & $0.91(2)$ & $\mathrm{C}(51)$ & $C(52)$ & $1.53(2)$ \\
\hline$C(53)$ & $C(54)$ & $1.455(8)$ & $C(54)$ & $C(55)$ & $1.45(1)$ \\
\hline$C(55)$ & $C(56)$ & $1.482(7)$ & $\mathrm{C}(57)$ & $\mathrm{C}(58)$ & $1.26(2)$ \\
\hline$C(57)$ & $C(62)$ & $1.37(1)$ & $\mathrm{C}(57)$ & $C(63)$ & $1.58(2)$ \\
\hline $\mathrm{C}(58)$ & $C(59)$ & $1.41(2)$ & $\mathrm{C}(59)$ & $\mathrm{C}(60)$ & $1.39(1)$ \\
\hline$C(60)$ & $C(61)$ & $1.36(1)$ & $\mathrm{C}(61)$ & $C(62)$ & $1.37(1)$ \\
\hline
\end{tabular}


Table S7. Bond Angles (deg) for 5.

\begin{tabular}{|c|c|c|c|c|c|c|c|}
\hline atom & atom & atom & angle & atom & atom & atom & angle \\
\hline $\mathrm{O}(1)$ & $\operatorname{Zr}(1)$ & $\mathrm{O}(2)$ & 109.9(1) & $\mathrm{O}(1)$ & $\operatorname{Zr}(1)$ & $\mathrm{O}(3)$ & $115.6(1)$ \\
\hline $\mathrm{O}(1)$ & $\operatorname{Zr}(1)$ & $\mathrm{O}(4)$ & $152.4(1)$ & $\mathrm{O}(1)$ & $\operatorname{Zr}(1)$ & $\mathrm{O}(5)$ & $77.2(1)$ \\
\hline $\mathrm{O}(1)$ & $\operatorname{Zr}(1)$ & $\mathrm{O}(6)$ & 84.31(9) & $\mathrm{O}(1)$ & $\operatorname{Zr}(1)$ & $\mathrm{C}(1)$ & $74.9(1)$ \\
\hline $\mathrm{O}(2)$ & $\operatorname{Zr}(1)$ & $\mathrm{O}(3)$ & $114.96(9)$ & $\mathrm{O}(2)$ & $\operatorname{Zr}(1)$ & $\mathrm{O}(4)$ & $85.63(9)$ \\
\hline $\mathrm{O}(2)$ & $\operatorname{Zr}(1)$ & $\mathrm{O}(5)$ & $152.22(9)$ & $\mathrm{O}(2)$ & $\operatorname{Zr}(1)$ & $\mathrm{O}(6)$ & $75.18(9)$ \\
\hline $\mathrm{O}(2)$ & $\operatorname{Zr}(1)$ & $\mathrm{C}(1)$ & $75.6(1)$ & $\mathrm{O}(3)$ & $\operatorname{Zr}(1)$ & $\mathrm{O}(4)$ & 74.61(9) \\
\hline $\mathrm{O}(3)$ & $\operatorname{Zr}(1)$ & $\mathrm{O}(5)$ & $83.08(9)$ & $\mathrm{O}(3)$ & $\operatorname{Zr}(1)$ & $\mathrm{O}(6)$ & $149.4(1)$ \\
\hline $\mathrm{O}(3)$ & $\operatorname{Zr}(1)$ & $\mathrm{C}(1)$ & $74.5(1)$ & $\mathrm{O}(4)$ & $\operatorname{Zr}(1)$ & $\mathrm{O}(5)$ & 78.91(9) \\
\hline $\mathrm{O}(4)$ & $\operatorname{Zr}(1)$ & $\mathrm{O}(6)$ & $77.75(9)$ & $\mathrm{O}(4)$ & $\operatorname{Zr}(1)$ & $\mathrm{C}(1)$ & $132.2(1)$ \\
\hline $\mathrm{O}(5)$ & $\operatorname{Zr}(1)$ & $\mathrm{O}(6)$ & 79.04(9) & $\mathrm{O}(5)$ & $\operatorname{Zr}(1)$ & $\mathrm{C}(1)$ & $131.5(1)$ \\
\hline $\mathrm{O}(6)$ & $\operatorname{Zr}(1)$ & $\mathrm{C}(1)$ & $135.3(1)$ & $\mathrm{C}(3)$ & $\mathrm{O}(1)$ & $\operatorname{Zr}(1)$ & $123.3(2)$ \\
\hline $\mathrm{C}(9)$ & $\mathrm{O}(2)$ & $\operatorname{Zr}(1)$ & $121.5(2)$ & $C(15)$ & $\mathrm{O}(3)$ & $\operatorname{Zr}(1)$ & $123.3(2)$ \\
\hline $\mathrm{C}(44)$ & $\mathrm{O}(4)$ & $\mathrm{C}(47)$ & $107.5(3)$ & $C(44)$ & $\mathrm{O}(4)$ & $\operatorname{Zr}(1)$ & $119.0(3)$ \\
\hline$C(47)$ & $\mathrm{O}(4)$ & $\operatorname{Zr}(1)$ & $124.0(2)$ & $\mathrm{C}(48)$ & $\mathrm{O}(5)$ & $\mathrm{C}(51)$ & $108.3(3)$ \\
\hline $\mathrm{C}(48)$ & $\mathrm{O}(5)$ & $\operatorname{Zr}(1)$ & $117.8(2)$ & $\mathrm{C}(51)$ & $\mathrm{O}(5)$ & $\operatorname{Zr}(1)$ & $123.7(3)$ \\
\hline$C(53)$ & $\mathrm{O}(6)$ & $C(56)$ & $107.6(3)$ & $C(53)$ & $\mathrm{O}(6)$ & $\operatorname{Zr}(1)$ & $126.8(3)$ \\
\hline$C(56)$ & $\mathrm{O}(6)$ & $\operatorname{Zr}(1)$ & $119.1(2)$ & $\mathrm{C}(2)$ & $\mathrm{C}(1)$ & $C(8)$ & $113.2(3)$ \\
\hline $\mathrm{C}(2)$ & $\mathrm{C}(1)$ & $\mathrm{C}(14)$ & 111.1(3) & $\mathrm{C}(2)$ & $\mathrm{C}(1)$ & $\operatorname{Zr}(1)$ & $108.0(2)$ \\
\hline $\mathrm{C}(8)$ & $\mathrm{C}(1)$ & $\mathrm{C}(14)$ & $110.0(3)$ & $\mathrm{C}(8)$ & $\mathrm{C}(1)$ & $\operatorname{Zr}(1)$ & $106.2(2)$ \\
\hline$C(14)$ & $\mathrm{C}(1)$ & $\operatorname{Zr}(1)$ & $108.1(2)$ & $\mathrm{C}(3)$ & $\mathrm{C}(2)$ & $\mathrm{C}(7)$ & $119.3(3)$ \\
\hline $\mathrm{C}(3)$ & $\mathrm{C}(2)$ & $\mathrm{C}(1)$ & $115.9(3)$ & $\mathrm{C}(7)$ & $\mathrm{C}(2)$ & $\mathrm{C}(1)$ & $124.5(3)$ \\
\hline C(4) & $\mathrm{C}(3)$ & $\mathrm{O}(1)$ & $123.4(3)$ & $\mathrm{C}(4)$ & $\mathrm{C}(3)$ & $\mathrm{C}(2)$ & $121.2(3)$ \\
\hline $\mathrm{O}(1)$ & $\mathrm{C}(3)$ & $\mathrm{C}(2)$ & $115.4(3)$ & $\mathrm{C}(5)$ & $\mathrm{C}(4)$ & $\mathrm{C}(20)$ & $120.6(3)$ \\
\hline$C(5)$ & $\mathrm{C}(4)$ & $\mathrm{C}(3)$ & $117.0(3)$ & $\mathrm{C}(20)$ & $\mathrm{C}(4)$ & $\mathrm{C}(3)$ & $122.3(3)$ \\
\hline$C(6)$ & $C(5)$ & $\mathrm{C}(4)$ & $123.8(4)$ & $\mathrm{C}(7)$ & $\mathrm{C}(6)$ & $\mathrm{C}(24)$ & $122.3(3)$ \\
\hline$C(7)$ & $C(6)$ & $C(5)$ & $117.0(4)$ & $C(24)$ & $C(6)$ & $C(5)$ & $120.7(3)$ \\
\hline $\mathrm{C}(2)$ & $C(7)$ & $C(6)$ & $121.6(3)$ & $\mathrm{C}(9)$ & $\mathrm{C}(8)$ & $\mathrm{C}(13)$ & $119.4(3)$ \\
\hline $\mathrm{C}(9)$ & $\mathrm{C}(8)$ & $\mathrm{C}(1)$ & $115.9(3)$ & $C(13)$ & $\mathrm{C}(8)$ & $\mathrm{C}(1)$ & $124.7(3)$ \\
\hline$C(10)$ & $\mathrm{C}(9)$ & $\mathrm{O}(2)$ & $123.4(3)$ & $C(10)$ & $\mathrm{C}(9)$ & $C(8)$ & $121.0(3)$ \\
\hline $\mathrm{O}(2)$ & $\mathrm{C}(9)$ & $\mathrm{C}(8)$ & $115.5(3)$ & $\mathrm{C}(11)$ & $C(10)$ & $\mathrm{C}(28)$ & $121.2(3)$ \\
\hline $\mathrm{C}(11)$ & $C(10)$ & $\mathrm{C}(9)$ & 116.1(3) & $C(28)$ & $C(10)$ & $\mathrm{C}(9)$ & $122.7(3)$ \\
\hline$C(12)$ & $\mathrm{C}(11)$ & $\mathrm{C}(10)$ & $124.2(4)$ & $C(13)$ & $C(12)$ & $C(32)$ & $122.2(4)$ \\
\hline$C(13)$ & $C(12)$ & $\mathrm{C}(11)$ & $117.0(3)$ & $C(32)$ & $C(12)$ & $\mathrm{C}(11)$ & $120.8(4)$ \\
\hline $\mathrm{C}(8)$ & $C(13)$ & $\mathrm{C}(12)$ & $122.1(4)$ & $C(15)$ & $C(14)$ & $\mathrm{C}(19)$ & $119.2(3)$ \\
\hline$C(15)$ & $C(14)$ & $\mathrm{C}(1)$ & $114.8(3)$ & C(19) & $C(14)$ & $\mathrm{C}(1)$ & $125.8(3)$ \\
\hline$C(16)$ & $C(15)$ & $\mathrm{O}(3)$ & $123.2(3)$ & $C(16)$ & $C(15)$ & $\mathrm{C}(14)$ & $120.9(3)$ \\
\hline $\mathrm{O}(3)$ & $C(15)$ & $\mathrm{C}(14)$ & $115.9(3)$ & $C(17)$ & $C(16)$ & $C(36)$ & $121.1(3)$ \\
\hline
\end{tabular}


Table S7. Bond Angles (deg) for 5. (Cont.)

\begin{tabular}{|c|c|c|c|c|c|c|c|}
\hline atom & atom & atom & angle & atom & atom & atom & angle \\
\hline$C(17)$ & $C(16)$ & $C(15)$ & $116.7(3)$ & $C(36)$ & $C(16)$ & $C(15)$ & $122.2(3)$ \\
\hline$C(18)$ & $\mathrm{C}(17)$ & $C(16)$ & $123.7(3)$ & $C(19)$ & $\mathrm{C}(18)$ & $\mathrm{C}(40)$ & $122.4(3)$ \\
\hline$C(19)$ & $\mathrm{C}(18)$ & $\mathrm{C}(17)$ & $117.3(3)$ & $\mathrm{C}(40)$ & $\mathrm{C}(18)$ & $\mathrm{C}(17)$ & $120.3(3)$ \\
\hline$C(14)$ & $\mathrm{C}(19)$ & $\mathrm{C}(18)$ & $122.1(3)$ & $\mathrm{C}(21)$ & $C(20)$ & $\mathrm{C}(22)$ & $109.2(4)$ \\
\hline $\mathrm{C}(21)$ & $\mathrm{C}(20)$ & $\mathrm{C}(23)$ & $107.2(4)$ & $\mathrm{C}(21)$ & $C(20)$ & $\mathrm{C}(4)$ & $112.8(4)$ \\
\hline$C(22)$ & $\mathrm{C}(20)$ & $\mathrm{C}(23)$ & $108.5(4)$ & $\mathrm{C}(22)$ & $\mathrm{C}(20)$ & $\mathrm{C}(4)$ & $109.4(3)$ \\
\hline$C(23)$ & $C(20)$ & $\mathrm{C}(4)$ & $109.6(4)$ & $C(25)$ & $C(24)$ & $C(26)$ & $111.4(5)$ \\
\hline$C(25)$ & $\mathrm{C}(24)$ & $\mathrm{C}(27)$ & $106.8(4)$ & $C(25)$ & $\mathrm{C}(24)$ & $C(6)$ & $110.1(4)$ \\
\hline$C(26)$ & $\mathrm{C}(24)$ & $\mathrm{C}(27)$ & $107.9(5)$ & $C(26)$ & $\mathrm{C}(24)$ & $C(6)$ & $109.1(4)$ \\
\hline$C(27)$ & $\mathrm{C}(24)$ & $C(6)$ & $111.5(4)$ & $\mathrm{C}(29)$ & $\mathrm{C}(28)$ & $C(30)$ & $108.2(4)$ \\
\hline$C(29)$ & $\mathrm{C}(28)$ & $\mathrm{C}(31)$ & $107.2(4)$ & $\mathrm{C}(29)$ & $\mathrm{C}(28)$ & $C(10)$ & $112.7(3)$ \\
\hline$C(30)$ & $\mathrm{C}(28)$ & $\mathrm{C}(31)$ & $108.0(4)$ & $C(30)$ & $\mathrm{C}(28)$ & $C(10)$ & $108.0(3)$ \\
\hline$C(31)$ & $\mathrm{C}(28)$ & $\mathrm{C}(10)$ & $112.7(3)$ & $C(33)$ & $C(32)$ & $C(34)$ & $108.2(4)$ \\
\hline$C(33)$ & $\mathrm{C}(32)$ & $C(35)$ & $106.1(4)$ & $C(33)$ & $C(32)$ & $\mathrm{C}(12)$ & $111.6(3)$ \\
\hline$C(34)$ & $\mathrm{C}(32)$ & $C(35)$ & $111.0(4)$ & $C(34)$ & $C(32)$ & $C(12)$ & $111.2(4)$ \\
\hline$C(35)$ & $\mathrm{C}(32)$ & $\mathrm{C}(12)$ & $108.6(3)$ & $C(37)$ & $C(36)$ & $\mathrm{C}(38)$ & $109.0(4)$ \\
\hline$C(37)$ & $C(36)$ & $\mathrm{C}(39)$ & $109.7(4)$ & $C(37)$ & $C(36)$ & $C(16)$ & $111.0(3)$ \\
\hline$C(38)$ & $C(36)$ & $\mathrm{C}(39)$ & $106.8(3)$ & $\mathrm{C}(38)$ & $C(36)$ & $C(16)$ & $112.3(3)$ \\
\hline C(39) & $\mathrm{C}(36)$ & $C(16)$ & $107.9(3)$ & $\mathrm{C}(41)$ & $\mathrm{C}(40)$ & $\mathrm{C}(42)$ & $108.7(4)$ \\
\hline$C(41)$ & $\mathrm{C}(40)$ & $\mathrm{C}(43)$ & $107.2(4)$ & $\mathrm{C}(41)$ & $\mathrm{C}(40)$ & $\mathrm{C}(18)$ & $108.8(4)$ \\
\hline$C(42)$ & $\mathrm{C}(40)$ & $\mathrm{C}(43)$ & $108.9(4)$ & $\mathrm{C}(42)$ & $\mathrm{C}(40)$ & $\mathrm{C}(18)$ & $110.3(3)$ \\
\hline$C(43)$ & $\mathrm{C}(40)$ & $\mathrm{C}(18)$ & $112.7(3)$ & $C(45)$ & $\mathrm{C}(44)$ & $\mathrm{O}(4)$ & $107.6(4)$ \\
\hline$C(46)$ & $\mathrm{C}(45)$ & $\mathrm{C}(44)$ & $106.0(5)$ & $\mathrm{C}(47)$ & $C(46)$ & $C(45)$ & $103.9(5)$ \\
\hline $\mathrm{O}(4)$ & $\mathrm{C}(47)$ & $C(46)$ & $105.7(4)$ & $C(49)$ & $\mathrm{C}(48)$ & $\mathrm{O}(5)$ & $106.8(4)$ \\
\hline$C(50)$ & C(49) & $C(52)$ & $34.5(8)$ & $\mathrm{C}(50)$ & $\mathrm{C}(49)$ & $\mathrm{C}(48)$ & 102.1(6) \\
\hline$C(52)$ & C(49) & $\mathrm{C}(48)$ & $108.4(7)$ & $\mathrm{C}(51)$ & $\mathrm{C}(50)$ & $C(52)$ & $76(1)$ \\
\hline$C(51)$ & $\mathrm{C}(50)$ & $\mathrm{C}(49)$ & $103.1(8)$ & $\mathrm{C}(49)$ & $C(50)$ & $C(52)$ & $68(1)$ \\
\hline$C(52)$ & $\mathrm{C}(51)$ & $\mathrm{O}(5)$ & $108.9(7)$ & $\mathrm{O}(5)$ & $\mathrm{C}(51)$ & $\mathrm{C}(50)$ & $103.9(7)$ \\
\hline$C(50)$ & $\mathrm{C}(51)$ & $C(52)$ & $35.3(8)$ & $\mathrm{C}(49)$ & $C(52)$ & $\mathrm{C}(50)$ & $77(1)$ \\
\hline$C(50)$ & $\mathrm{C}(52)$ & $\mathrm{C}(51)$ & $68(1)$ & $\mathrm{C}(51)$ & $C(52)$ & $\mathrm{C}(49)$ & $103.2(8)$ \\
\hline$C(54)$ & $C(53)$ & $\mathrm{O}(6)$ & $106.2(4)$ & $C(55)$ & $C(54)$ & $\mathrm{C}(53)$ & $105.3(5)$ \\
\hline$C(56)$ & $\mathrm{C}(55)$ & $\mathrm{C}(54)$ & $105.4(5)$ & $\mathrm{O}(6)$ & $C(56)$ & $C(55)$ & $106.2(4)$ \\
\hline$C(58)$ & $\mathrm{C}(57)$ & $C(62)$ & $124(1)$ & $\mathrm{C}(58)$ & $C(57)$ & $C(63)$ & $119.0(8)$ \\
\hline$C(62)$ & $\mathrm{C}(57)$ & $\mathrm{C}(63)$ & 116.1(9) & $\mathrm{C}(59)$ & $\mathrm{C}(58)$ & $C(57)$ & $120(1)$ \\
\hline$C(60)$ & $\mathrm{C}(59)$ & $\mathrm{C}(58)$ & $117(1)$ & $C(61)$ & $C(60)$ & $\mathrm{C}(59)$ & $120.3(9)$ \\
\hline$C(62)$ & $\mathrm{C}(61)$ & $\mathrm{C}(60)$ & $119.9(7)$ & $C(57)$ & $C(62)$ & $\mathrm{C}(61)$ & $117.7(8)$ \\
\hline
\end{tabular}


Figure S4. Kinetics plots of the isomerization of $\mathbf{1 a}$ into $\mathbf{1 b}$ in toluene- $d_{8}$.
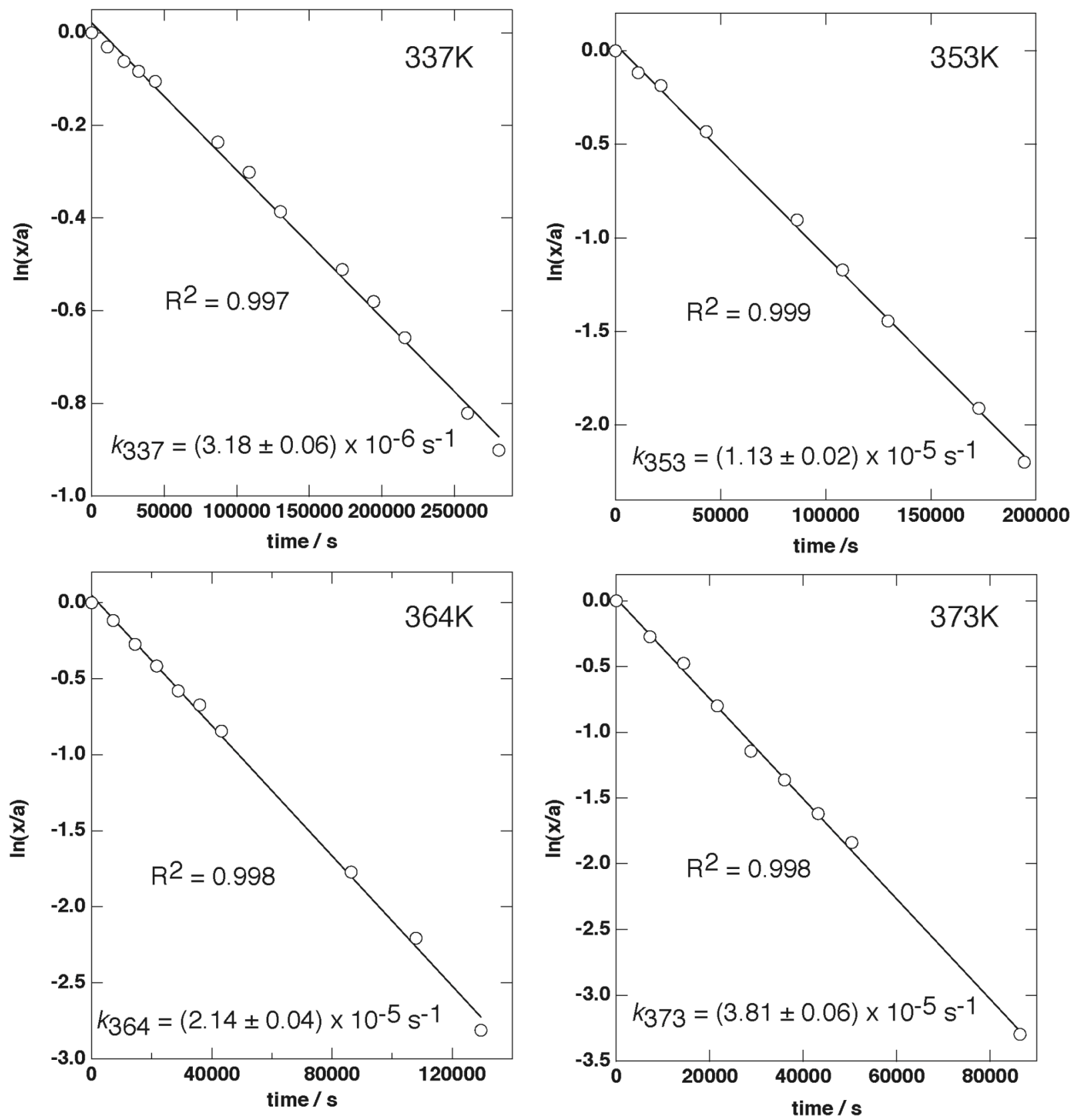
Figure S5. Kinetics plots of the isomerization of $\mathbf{1 a}$ into $\mathbf{1 b}$ in 1,2-dichlorobenzene- $d_{4}$.
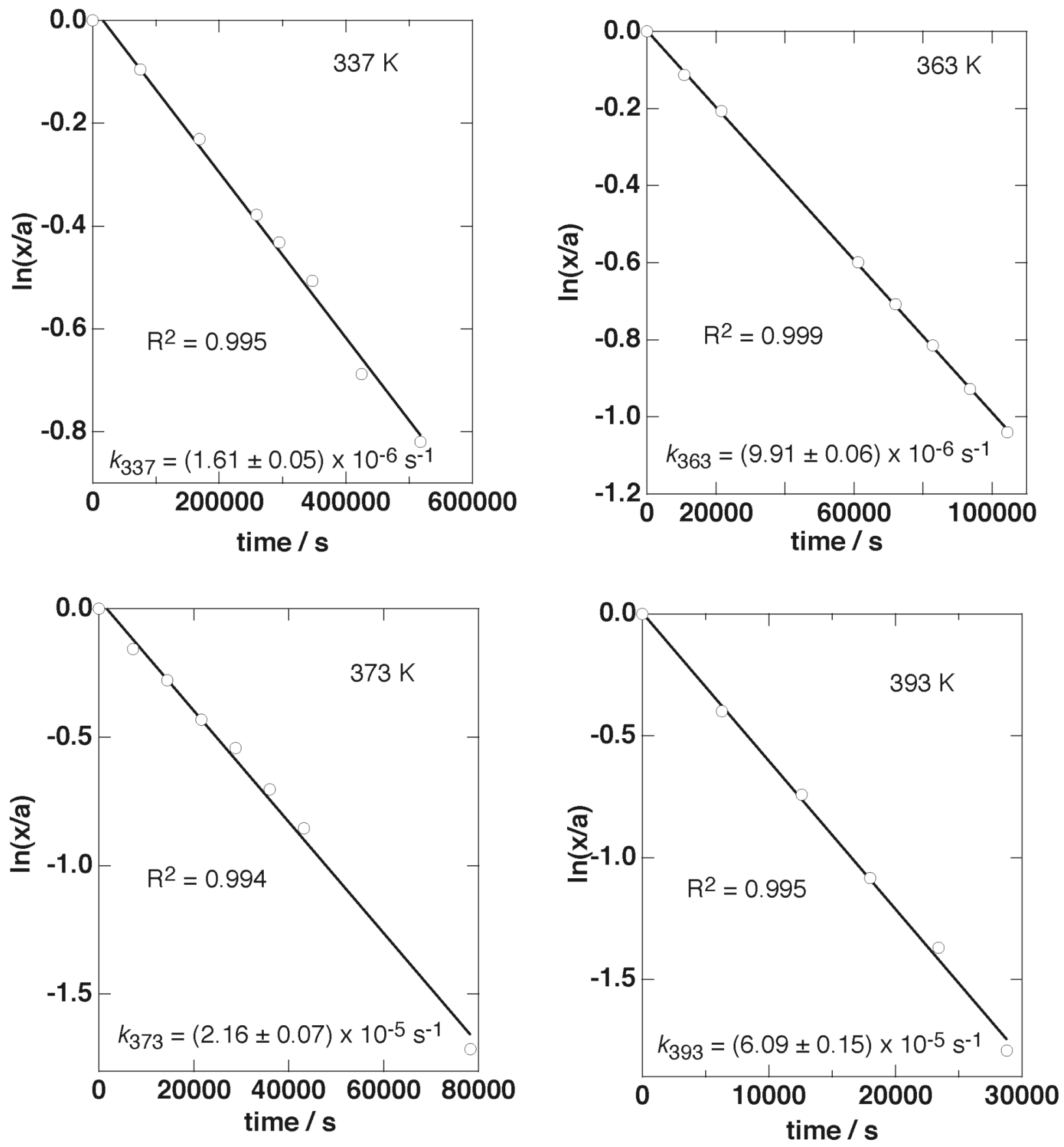
Figure S6. Arrhenius plot for the isomerization of $\mathbf{1 a}$ into $\mathbf{1 b}$ in toluene- $d_{8}$ (A) and in 1,2-dichlorobenzene- $d_{4}$ (B). Extracted Activation Parameters are as follows; in toluene- $d_{8}, \Delta H^{*}=$ $16.3(4) \mathrm{kcal} / \mathrm{mol}$ and $\Delta S^{\ddagger}=-35(1) \mathrm{cal} /(\mathrm{mol} \mathrm{K})$; in 1,2-dichlorobenzene- $d_{4}, \Delta H^{\ddagger}=16.5(5) \mathrm{kcal} / \mathrm{mol}$ and $\Delta S^{\ddagger}=-36(2) \mathrm{cal} /(\mathrm{mol} \mathrm{K})$.
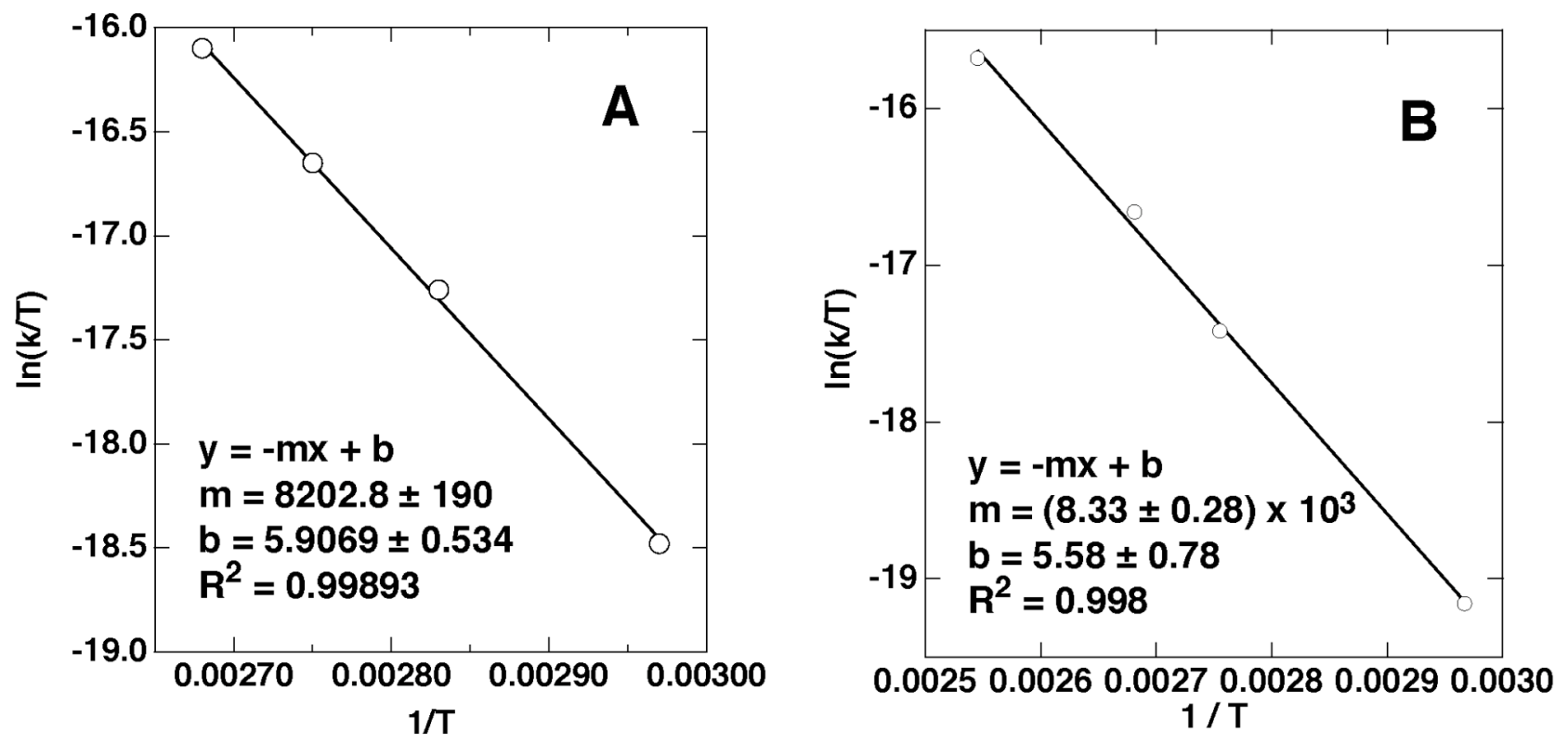